\title{
Article \\ Automatic Measurement of Morphological Traits of Typical Leaf Samples
}

\author{
Xia Huang ${ }^{1,2} \mathbb{1}$, Shunyi Zheng ${ }^{1,2, *}$ and Li Gui ${ }^{1,2}$ \\ 1 School of Remote Sensing and Information Engineering, Wuhan University, Wuhan 430079, China; \\ yuxiuhx@whu.edu.cn (X.H.); whuguili@whu.edu.cn (L.G.) \\ 2 Collaborative Innovation Center of Geospatial Technology, Wuhan 430079, China \\ * Correspondence: syzheng@whu.edu.cn; Tel.: +1-872-306-8326
}

check for

updates

Citation: Huang, X.; Zheng, S.; Gui, L. Automatic Measurement of Morphological Traits of Typical Leaf Samples. Sensors 2021, 21, 2247. https://doi.org/10.3390/s21062247

Academic Editor: Thomas Udelhoven

Received: 4 February 2021

Accepted: 19 March 2021

Published: 23 March 2021

Publisher's Note: MDPI stays neutral with regard to jurisdictional claims in published maps and institutional affiliations.

Copyright: (c) 2021 by the authors. Licensee MDPI, Basel, Switzerland. This article is an open access article distributed under the terms and conditions of the Creative Commons Attribution (CC BY) license (https:// creativecommons.org/licenses/by/ $4.0 /)$.
Abstract: It is still a challenging task to automatically measure plants. A novel method for automatic plant measurement based on a hand-held three-dimensional (3D) laser scanner is proposed. The objective of this method is to automatically select typical leaf samples and estimate their morphological traits from different occluded live plants. The method mainly includes data acquisition and processing. Data acquisition is to obtain the high-precision 3D mesh model of the plant that is reconstructed in real-time during data scanning by a hand-held 3D laser scanner (ZGScan 717, made in Zhongguan Automation Technology, Wuhan, China). Data processing mainly includes typical leaf sample extraction and morphological trait estimation based on a multi-level region growing segmentation method using two leaf shape models. Four scale-related traits and six corresponding scale-invariant traits can be automatically estimated. Experiments on four groups of different canopy-occluded plants are conducted. Experiment results show that for plants with different canopy occlusions, $94.02 \%$ of typical leaf samples can be scanned well and $87.61 \%$ of typical leaf samples can be automatically extracted. The automatically estimated morphological traits are correlated with the manually measured values EF (the modeling efficiency) above 0.8919 for scale-related traits and EF above 0.7434 for scale-invariant traits). It takes an average of 196.37 seconds (186.08 seconds for data scanning, 5.95 seconds for 3D plant model output, and 4.36 seconds for data processing) for a plant measurement. The robustness and low time cost of the proposed method for different canopyoccluded plants show potential applications for real-time plant measurement and high-throughput plant phenotype.

Keywords: plant measurement; morphological traits; 3D plant model; segmentation

\section{Introduction}

Leaf morphological traits are indices describing plant shape and architecture. They can be used in many fields of agriculture research, such as plant growth and health condition monitoring [1], plant modeling [2], agricultural simulation [3], farm management decision-making design [4], high-throughput plant phenotype analysis [5], and genomewide association study [6]. The sample leaves are mainly selected based on experience in traditional leaf trait measurement, which is time-consuming and cumbersome. In some agricultural research, such as high-throughput phenotype, a large number of samples are needed [7]. The automatic sample selection has become necessary. Nevertheless, various sensors, such as the color digital cameras, range cameras, hyperspectral cameras, multispectral cameras, thermal imagers, infrared radiometers, fluorescence sensors, light detection and ranging (LIDAR) sensors, global positioning system (GPS) receivers, and laser sensors have been used for plant measurement [8]. Those sensors can generate a large amount of data. Traditional leaf trait measurement that mainly relies on manual measurement methods based on contact tools is not suitable for such a large capacity of data processing. Automatic morphological trait estimation should be carried out and automatic 
data batch processing is urgently needed. Moreover, the leaf morphological structures of different species are different, which makes the calculation of leaf morphological traits vary. Therefore, it is significant to study automatic measurement of morphological traits of typical leaf samples.

Image technology has been widely used in plant measurement. An et al. [9] developed an imaging system and proposed a series of image processing algorithms to construct two-dimensional (2D) mosaicked orthophotos. Morphological traits such as leaf length and rosette area were measured by these 2D top-view images. Fanourakis et al. [10] used a phenotypic platform light curtain array (LC) to evaluate leaf area and maximum height from side-view profile images. Pereyrairujo et al. [11] introduced a low-cost platform for high-throughput measurement of plant growth. This imaging approach estimated morphological traits based on 2D images. The top-view and side-view images were required to ensure measurement accuracy when measuring leaf morphological traits such as area, perimeter, length, and width. If the image was shot at random angles, the measurement results would deviate greatly from the true values.

Since the aforementioned works based on 2D imaging technology not only require images with special shooting angles but also lose part of the 3D information, 3D technology that can accurately measure the 3D structure of plants is rising rapidly. Biskup et al. [12] used an area-based binocular stereo imaging system to build a 3D plant model and measure leaf inclination angles. Gibbs et al. [13] adopted the structure-from-motion (SFM) technique to build a 3D plant model. This method required a large number of images and cost a lot of time to reconstruct the 3D model. Kjaer et al. [14] employed a 3D NIR-laser scanner to track daily changes in the growth stages of different plants in challenging environments. The scanner was fixed and the 3D model of the plant was incomplete. Xia et al. [15] used a Kinect RGB-D camera to measure individual plant leaves. Mean shift clustering was employed to segment individual leaves from the background. However, no morphological traits were estimated. Drapikowski [16] employed a structured-light DAVID to estimate the morphological traits of Xerophytic plants. The morphological traits such as length, width, and area were automatically estimated based on rough plant models. Martínezguanter et al. [17] used a LiDAR sensor to detect plant spacing. A large number of 3D models of plants were reconstructed. The point cloud data obtained by Kinect, David, and LiDAR were usually rough, so it was difficult to reconstruct high-precision $3 \mathrm{D}$ plant models. These methods usually obtained 3D points first, and then the 3D plant model was reconstructed to measure morphological traits. However, these models were usually very rough and lacked detailed geometry information, which would affect the measurement accuracy. Thus, it is significant to obtain high-precision 3D models of plants.

Furthermore, most contemporary studies of leaf trait measurement are mostly limited to the plants without canopy occlusion at the early growth stage, and all leaves are counted $[18,19]$. Plants with heavy occlusion are rarely explored in individual leaf trait measurement research. More leaves mean heavier occlusion problems, which makes adequate data acquisition difficult and automated data analysis challenging. However, canopy occlusion is common in reality. Therefore, it is practically significant to measure individual leaf morphological traits of plants with different canopy occlusions. Additionally, most contemporary research of leaf measurement involves all leaves on the plants. In research such as leaf classification and plant species identification, typical leaf samples do not include the curved new-born leaves, damaged leaves, or rotten leaves [20,21]. Therefore, it is worthwhile to study the automatic selection of typical leaf samples. Nevertheless, the morphological structures of leaves are different. Specifically, leaves of plants, such as corn [22-24], rice [25], and wheat [26], are long and banding. Leaves of plants, such as Epipremnum aureum [27], Anthurium Andraeanum [28], and leafy vegetables [29], are broad and elliptical. The calculation algorithms of their morphological traits, especially length and width, are different. Additionally, leaf morphological traits in the aforementioned research are usually leaf area, length, and width. Those traits are scale-related. More scale-invariant traits should be explored. 
Thus, research of new methods that employs 3D technology for automatic plant measurement is of great interest. The automatic data acquisition and processing methods that can perform high-throughput phenotyping functions with adequate precision requirements are useful in modern agriculture. The objective of this study is to solve three problems, namely, the acquisition of the high-precision 3D plant model, automatic selection of typical leaf samples, and automatic estimation of leaf morphological traits. In this paper, we propose a novel method to automatically select typical leaf samples and estimate their morphological traits from different canopy-occluded plants based on a hand-held 3D laser scanner. The method mainly includes two parts: (1) data acquisition with a hand-held 3D laser scanner to obtain the 3D mesh model of the plant, and (2) the multi-level region growing segmentation using two leaf shape models to automatically select typical leaf samples and estimate their morphological traits. Particularly, the 3D plant model can be reconstructed in real-time during data scanning. When segmenting individual leaves of different plants at different scales, the two main segmentation parameters, smooth and curvature, are adaptive. Two shape models, one using the scale-related traits and the other employing the scale-invariant traits, are dynamically established based on the segmentation results at different scales using the principal component analysis method. Four scale-related morphological traits and six corresponding scale-invariant traits are automatically estimated. Epipremnum aureum, one of the most common plants with broad and elliptical leaf structure, is adopted as the experiment subject. The proposed method will be tested on four groups of Epipremnum aureum with different canopy occlusions. The accuracies of the data scanning, segmentation, and trait estimation will be analyzed.

\section{Materials and Methods}

\subsection{Data Acquisition and Processing Environment}

The data acquisition was performed using a ZGScan 717 (parameters listed in Table 1) hand-held 3D laser scanner in Wuhan, China. The time was November 2020. The data acquisition was conducted indoors. There were four groups of Epipremnum aureum with different canopy occlusions. Each group contained three plants. Plants in each group had similar canopy occlusion. The occlusion increased from the first group to the fourth group. Specifically, the plants of the first, second, third, and fourth groups had no, a little, medium, and heavy canopy occlusion. The plants in each group were scanned individually.

Table 1. Parameters of ZGScan 717.

\begin{tabular}{cccc}
\hline Types & Parameters & Types & Parameters \\
\hline Weight & $1.0 \mathrm{~kg}$ & Accuracy & $0.03 \mathrm{~mm}$ \\
Volume & $310 \times 147 \times 80 \mathrm{~mm}$ & Field depth & $550 \mathrm{~mm}$ \\
Scanning area & $275 \times 250 \mathrm{~mm}$ & Transfer method & USB 3.0 \\
Speed & 480,000 times $/ \mathrm{s}$ & Work temperatures & $-20-40{ }^{\circ} \mathrm{C}$ \\
Light & 7 laser crosses $(+1)$ & Work humidity & $10-90 \%$ \\
Light security & II & Outputs & Point clouds $/ 3 \mathrm{D}$ \\
\end{tabular}

The plant was on the table and scanned by ZGScan 717 (Figure 1a). With two CCDs and one laser, ZGScan 717 recorded data on a contour section of an object surface by projecting seven pairs of cross scanning laser beams and one deep hole scanning laser beam. To reconstruct the 3D model of the plant, the position and orientation of the detection handle were detected by the embedded motion-tracking technology. It quickly scanned the object (480,000 times/second) and generated high-precision point clouds. The maximum accuracy could reach $0.03 \mathrm{~mm}$. ZGScan 717 was held close to the plant during data scanning with no closer than $80 \mathrm{~mm}$ and no distant than $2000 \mathrm{~mm}$ to ensure a high-resolution scanning result. The scanning path was random. The 3D model was reconstructed in real-time when data scanning. Particularly, the reconstructed 3D model was viewed on a laptop in real-time when data scanning, which helped to check the integrity of the data acquisition. 
Specifically, complex parts of the plant should be scanned more carefully. Particularly, the software integrated with ZGScan 717 could output both 3D point clouds and 3D mesh. Here, the 3D mesh was chosen as the scanning output (Figure 1b). Figure 1 shows the data acquisition process. As shown in Figure 1b,c, the output 3D mesh model of the plant is high-precision with detailed geometric structure.
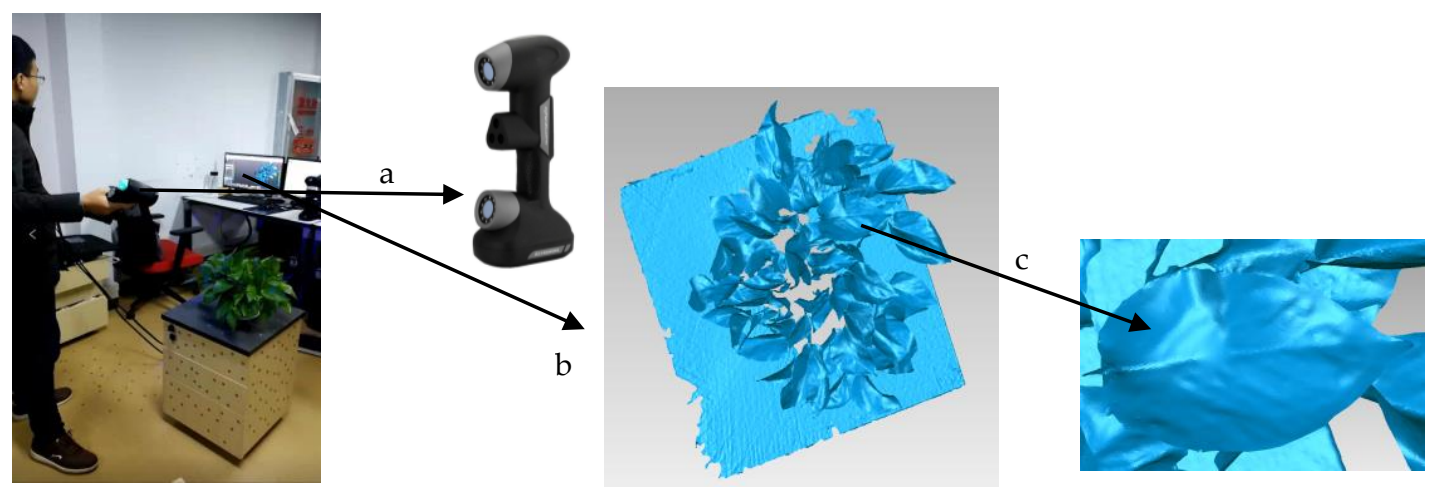

Figure 1. Data acquisition process. (a) ZGScan 717; (b) The generated 3D mesh model of the plant; (c) The 3D mesh model of a leaf.

The data processing algorithms were implemented on a $2.50 \mathrm{GHZ}$ desktop with 8.0 Gb RAM. The code was compiled using C++ with Point Cloud Library (PCL) v1.8.0, Computational Geometry Algorithms Library (CGAL), and Visualization Toolkit (VTK) v7.0. All algorithms were integrated. The entire process of data processing was conducted by algorithms without manual works.

\subsection{Typical Leaf Sample Extraction and Morphological Trait Estimation}

Individual leaves should be segmented to measure leaf morphological traits. A multilevel region growing segmentation method based on the leaf shape model is proposed. The region growing method divides the point clouds into different clusters mainly based on smooth and curvature characters [30]. Points with similar smooth and curvature values are segmented into a group. Different smooth and curvature thresholds can lead to different results. Segmentation at a large scale based on the region growing method will lead to a rough segmentation result, and if the segmentation scale is too small, it may be oversegmented. When the input data varies, the most appropriate scale should be different. However, the segmentation scale and its parameters are usually set by experience in most contemporary research. The goal of our proposed segmentation method is to find a suitable adaptive segmentation scale for different input data. Considering that leaves in the same species have similar morphological structures, the shape model is employed in segmentation. The proposed segmentation method mainly includes six steps, as shown in Figure 4.

Step 1: Non-plant removal. The main purpose of non-plant removal is to remove the plant from the background. Here, the RANSAC [31] plane detection method is adopted to remove the table, as shown in Figure $2 \mathrm{~b}$. Then we filter out the triangles with a distance range of $\varepsilon(\varepsilon=100 \mathrm{~mm})$ from the table, as shown in Figure 2c. Those data are usually the table, flower pot, and the soil. At last, plant data are extracted from the background (Figure 2d). Particularly, some data of the flower pot and the soil may be missing in some experiments, as they are not our target and are not scanned properly during the scanning process, as shown in Figure 2. 


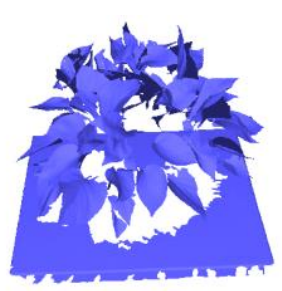

(a)

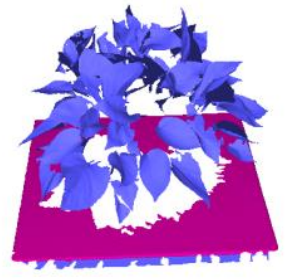

(b)

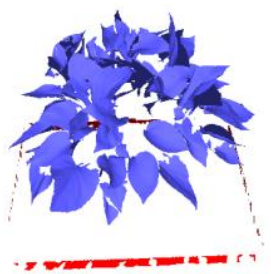

(c)

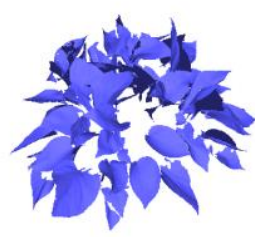

(d)

Figure 2. The removal of non-plant. (a) An example of the generated 3D mesh model of the plant; (b) the detection of the table; (c) the filtering of non-plant; (d) the plant after the non-plant removal.

Step 2: Region growing segmentation. Smooth and curvature are two decisive parameters for region growing segmentation. The most important step is to find the appropriate values of smooth and curvature. The average of the smooth and curvature, $\zeta$ and $\rho$, are calculated first. The number of points $N_{p}$ for fitting a local surface and calculating curvature is usually 30 . The values of smoothness and curvature thresholds $\varepsilon_{\mathrm{a}}$ and $\varepsilon_{\mathrm{b}}$ in the initial segmentation scale are $\varepsilon_{\mathrm{a}}=1.5 \varsigma$ and $\varepsilon_{\mathrm{b}}=1.5 \rho$.

Step 3: Estimation of morphological traits. Assume all the segmentation results are leaves. All morphological traits of each segmentation result are calculated. Typical shapebased leaf morphological traits include scale-related and scale-invariant traits. Generally, leaf area, perimeter, length, and width are scale-related morphological traits, and their ratios are scale-invariant morphological traits, as listed in Table 2.

Table 2. Morphological traits. Sym.: symbols of the traits. Var.: variables.

\begin{tabular}{cccccc}
\hline Scale-Related Traits & Sym. & Var. & Scale-Invariant Traits & Sym. & Var. \\
\hline Area & $s$ & $X_{01}$ & Area perimeter ratio & $s / c$ & $X_{11}$ \\
Perimeter & $c$ & $X_{02}$ & Area length ratio & $s / l$ & $X_{12}$ \\
Length & $l$ & $X_{03}$ & Area width ratio & $s / w$ & $X_{13}$ \\
Width & $w$ & $X_{04}$ & Perimeter length ratio & $c / l$ & $X_{14}$ \\
& & & Perimeter width ratio & $c / w$ & $X_{15}$ \\
& & & Aspect ratio & $l / w$ & $X_{16}$ \\
\hline
\end{tabular}

Leaf area (s) is an important scale-related trait. As shown in Figure 3, the leaf area can be represented by a triangular mesh (green mesh). Leaf perimeter $(c)$ is the boundary of the mesh (blue line in Figure 3). The length of the boundary is the leaf perimeter. Leaf length $(l)$ is usually measured along the midline of the leaf. The shape of the leaf is broad and elliptical; thus, the longest shortest geodesic path along the first principal component direction (purple line in Figure 3) is the target leaf length (red line in Figure 4). Leaf width $(w)$ (yellow line in Figure 3 ) is usually defined as the widest length of the leaf perpendicular to the leaf length.

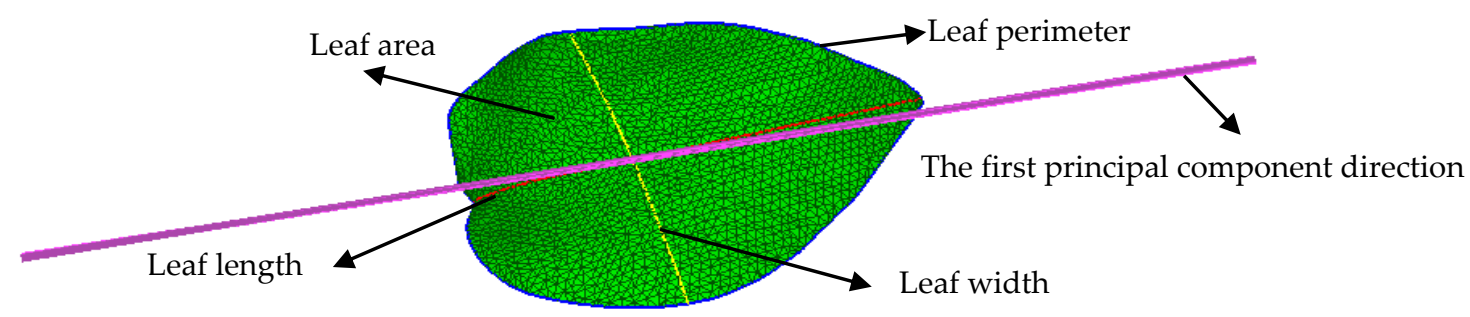

Figure 3. Visualization of four scale-related morphological traits (leaf area, perimeter, length, and width) on the 3D-triangle mesh of a leaf. 


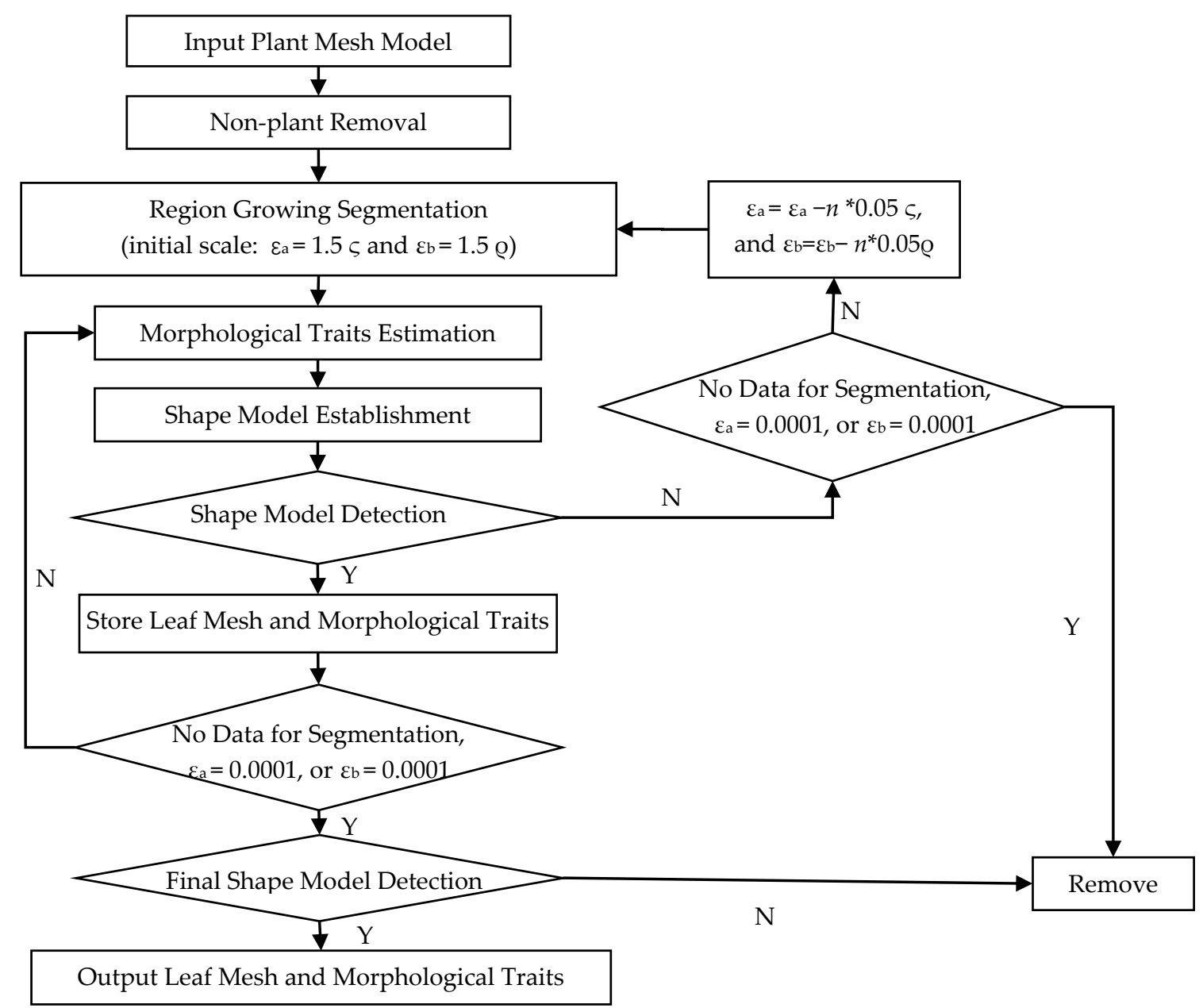

Figure 4. The multi-level region growing segmentation method based on the shape model.

Step 4: Establishment of the shape model. Two shape models are established to describe the leaf parametrically. One is used for size description and the other is employed for architecture description. The formulas for these two shape models are as follows:

$$
\begin{gathered}
F(X)=a_{1} \frac{X_{01}-\overline{X_{01}}}{\overline{X_{01}}}+a_{2} \frac{X_{02}-\overline{X_{02}}}{\overline{X_{02}}}+a_{3} \frac{X_{03}-\overline{X_{03}}}{\overline{X_{03}}}+a_{4} \frac{X_{04}-\overline{X_{04}}}{\overline{X_{04}}} \\
G(X)=b_{1} X_{11}+b_{2} X_{12}+b_{3} X_{13}+b_{4} X_{14}+b_{5} X_{15}+b_{6} X_{16}
\end{gathered}
$$

where $a_{1}-a_{4}$ and $b_{1}-b_{6}$ are variance contribution rates of the principal component analysis of the scale-related traits and scale-invariant traits, respectively. $X_{01} \sim X_{04}$ and $X_{11} \sim X_{16}$ are traits in Table 2.

Step 5: Detection of the shape model. A series of clusters are obtained after the initial segmentation. Some leaves can be successfully segmented, while some attached and overlapped leaves may be segmented incorrectly. Therefore, correct results should be stored, and incorrect clusters should be further segmented. If a segmented result satisfies:

$$
H(X)=\left\{\begin{array}{c}
0.25 * \overline{F(X)}<F(X)<1.25 * \overline{F(X)} \\
0.25 * \overline{G(X)}<G(X)<1.25 * \overline{G(X)}
\end{array}\right.
$$

The segmented result is a typical leaf sample, and its mesh and morphological traits are stored. If not, repeat step 2 with a smaller segmentation scale (smaller values of $\varepsilon_{\mathrm{a}}$ and $\left.\varepsilon_{\mathrm{b}}\right) . \varepsilon_{\mathrm{a}}$ and $\varepsilon_{\mathrm{b}}$ are reduced by $5 \%$ in each cycle. Repeat this process until all segmentation results meet the shape model detection or one of $\varepsilon_{\mathrm{a}}$ and $\varepsilon_{\mathrm{b}}$ is reduced to 0.0001 . 
Step 6: Final shape model detection and the outputs. When the segmentation is done, detect all the stored segmentation results with the final shape model that is based on all the stored segmentation results. If a segmentation result satisfies the final shape model, the 3D leaf model and their morphological traits are output.

The multi-scale segmentation method proposed in this paper is an iterative process. Rough segmentation results $C_{\text {level1 }}$ are usually obtained through the initial segmentation $\left(\varepsilon_{\mathrm{a}}=1.5 \mathrm{c}\right.$ and $\left.\varepsilon_{\mathrm{b}}=1.5 \rho\right)$, as shown in Figure $5 \mathrm{a}$. The first shape model $\mathrm{H}(\mathrm{X}) \_$level1 is built based on all clusters in $\mathrm{C}_{-}$level1. Then all clusters in $\mathrm{C}_{-}$level1 are detected by $\mathrm{H}(\mathrm{X})$ _level1. Clusters in $C_{-}$level1 that satisfy the $\mathrm{H}(\mathrm{X})$ _level1, defined as $\mathrm{M}_{-} \mathrm{H}(\mathrm{X})$ _level1 , are stored, as shown in Figure 5d. Clusters that do not satisfy the $\mathrm{H}(\mathrm{X})$ _level1, defined as $\mathrm{M}_{-} \mathrm{R}_{-}$level1 , go through the second segmentation at a smaller scale $\left(\varepsilon_{\mathrm{a}}=1.45 \mathrm{~s}\right.$ and $\left.\varepsilon_{\mathrm{b}}=1.45 \rho\right)$, as shown in Figure $5 \mathrm{~b}$. Then a new shape model $\mathrm{H}(\mathrm{X})$ _level2 is built based on $\mathrm{M}_{-} \mathrm{H}(\mathrm{X})$ _level1 and the second segmentation results $\mathrm{C}_{-}$level2. Clusters in $\mathrm{M}_{-} \mathrm{H}(\mathrm{X})$ _level1 and $\mathrm{C}_{-}$level2 that satisfy the $\mathrm{H}(\mathrm{X})$ _level2, defined as $\mathrm{M}_{-} \mathrm{H}(\mathrm{X})_{-}$level2, update $\mathrm{M}_{-} \mathrm{H}(\mathrm{X})_{-}$level1, as shown in Figure 5e. Clusters in $\mathrm{C}_{-}$level2 that do not satisfy the $\mathrm{H}(\mathrm{X})$ _level2, define as $\mathrm{M}_{\_} \mathrm{R}_{-}$level2, go through further segmentation, as shown in Figure 5c. The segmentation continues until all segmentation results meet the shape model detection or one of $\varepsilon_{\mathrm{a}}$ and $\varepsilon_{\mathrm{b}}$ is reduced to 0.0001 . $\mathrm{M}_{-} \mathrm{H}(\mathrm{X}) \_$leveln is defined as the clusters that satisfy the last shape model $\mathrm{H}(\mathrm{X})$ _leveln after the final segmentation. When the segmentation is done, a final shape model $H(X)$ is built based on clusters in $\mathrm{M}_{\_} \mathrm{H}(\mathrm{X})_{-}$leveln . All clusters in $\mathrm{M}_{-} \mathrm{H}(\mathrm{X})_{\_}$leveln are detected by $\mathrm{H}(\mathrm{X})$. Then clusters $\left(\mathrm{M}_{-} \mathrm{H}(\mathrm{X})\right.$ ) that satisfy the $H(X)$ are the typical leaf samples, as shown in Figure 5f. As more and more typical leaves are segmented correctly, the established shape model becomes more and more accurate, which in turn improves the automatic segmentation accuracy of typical leaves. A segmentation result may be considered as the typical leaf sample after the initial segmentation based on the initial shape model, while it will be removed in further shape model detection, as shown in the red box in Figure 5.
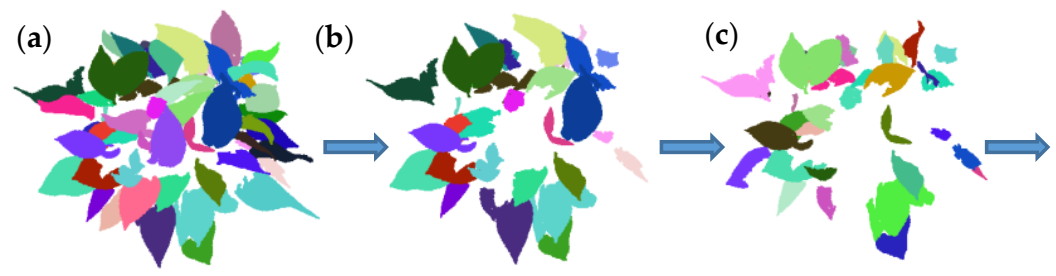

\section{Segmentation continues}

(d)

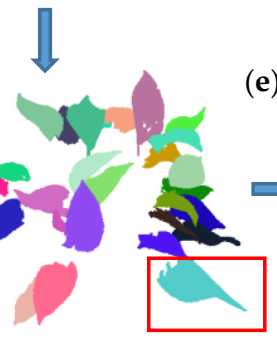

(e)

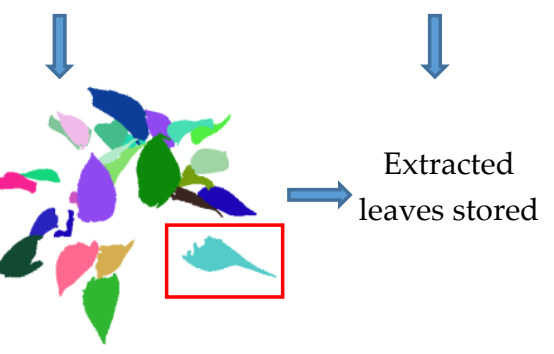

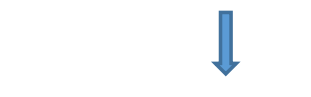

(f)

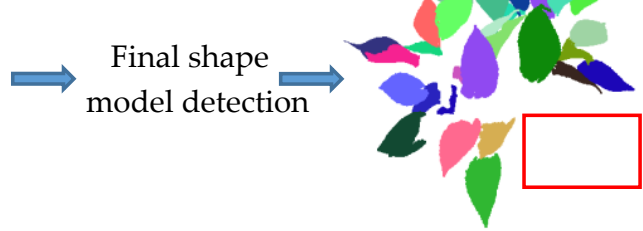

Figure 5. Schematic diagram of the multi-level region growing segmentation. (a) Initial segmentation results $\left(\varepsilon_{\mathrm{a}}=1.5 \varsigma\right.$ and $\left.\varepsilon_{\mathrm{b}}=1.5 \rho\right)$; (b) the data for the second segmentation; (c) the data for the third segmentation; (d) the automatically selected leaves after the initial segmentation based on the initial shape models; (e) the automatically selected leaves after the second segmentation $\left(\varepsilon_{\mathrm{a}}=1.45 \varsigma\right.$ and $\left.\varepsilon_{\mathrm{b}}=1.45 \rho\right)$ based on the second shape models; $(\mathrm{f})$ the automatically selected typical leaf samples after the final segmentation based on the final shape models. The red boxes mark a cluster considered as a typical leaf sample during the multi-level segmentation process but removed at last.

\subsection{Accuracy Analysis}

The accuracies of the data scanning, segmentation, and trait estimation are tested. The comparison between automatically and manually selected typical leaf samples is conducted to illustrate the accuracy of the segmentation. $R_{-}$scan is the data scanning accuracy. $R_{-} \operatorname{seg} 1$ is the segmentation accuracy between automatically segmented typical leaf samples and well- 
scanned typical leaf samples. $R_{-}$seg 2 is the segmentation accuracy between automatically segmented typical leaf samples and manually selected typical leaf samples. $R_{-}$scan,$R_{-}$seg 1 , and $R_{-}$seg 2 can be calculated as follows:

$$
R_{\_s c a n}=\frac{N_{2}}{N_{1}} \times 100 \%, R_{\_s e g 1}=\frac{N_{3}}{N_{2}} \times 100 \%, \text { and } R_{s e g 2}=\frac{N_{3}}{N_{1}} \times 100 \%
$$

where $N_{1}, N_{2}$, and $N_{3}$ are the numbers of manually selected, well scanned, and automatically selected typical leaf samples.

Correlation analyses between the automatically estimated and manually measured traits are performed to verify the accuracy of trait estimation. A dimensionless statistic that directly relates model predictions to observed data is the modeling efficiency $(E F)$ [32]. $E F$ is a statistic based on the coefficient of determination. The calculated $E F$ is an overall indication of goodness of fit. If a model has a negative value of $E F$, the model cannot be recommended. If $E F$ has preferable values close to one, it indicates a "near-perfect" model. $E F$ is defined as:

$$
E F=1-\sum_{i=1}^{n}\left(y_{i}-x_{i}\right)^{2} / \sum_{i=1}^{n}\left(y_{i}-\bar{y}\right)^{2}
$$

where $x_{i}$ is the independent variable, $y_{i}$ is the dependent variable, and $n$ is the number of samples. In this paper, $x_{i}$ is the automatically estimated morphological trait and $y_{i}$ is the manually measured morphological trait.

Additionally, the root-mean-square error (RMSE) and the mean absolute percentage error (MAPE) of the automatic measurement traits compared to the manually measured traits are adopted to illustrate the accuracy of trait estimation. The formulas for RMSE and MAPE are as follows:

$$
\begin{gathered}
R M S E=\sqrt{\frac{1}{n} \sum_{i=1}^{n}\left(x_{m i}-x_{a i}\right)^{2}} \\
M A P E=\frac{1}{n} \sum_{i=1}^{n} \frac{\left|x_{m i}-x_{a i}\right|}{x_{m i}} \times 100 \%
\end{gathered}
$$

where $x_{m i}$ is the manual measurement traits and $x_{a i}$ is the automatically estimated traits.

\section{Results}

\subsection{Results of Data Scanning and Segmentation}

The data scanning results and automatic segmentation results of typical leaf samples are shown in Table 3. The number of leaves $N_{0}$ in the first and second group is 3-8 and 10-11. The number of typical leaf samples $N_{1}$ in the first and second group is 3-6 and $8-11$. The difference between $N_{0}$ and $N_{1}$ indicates that there are some new-born leaves or damaged leaves that are not suitable for morphological trait estimation. The scanning accuracy $\left(R_{-}\right.$scan $)$and segmentation accuracies $\left(R_{-s e g}\right.$ and $R_{-}$seg2 $)$reach $100 \%$. This indicates the data scanning accuracy with the hand-held scanner ZGScan 717 is competitive and the automatic segmentation method proposed in this paper is effective for plants with no or a little occlusion. For plants in the third group, $N_{0}$ ranges from 26 to 34 , and $N_{1}$ varies from 24 to 31. This indicates there are new-born or damaged leaves. The values of $R_{-}$scan (96.77-95.83\%), R_seg1 (95.83-93.33\%), and R_seg2 (92.00-90.32\%) exhibit that the proposed segmentation method is practical for the plants with medium occlusion, although some data are missing. In the fourth group, $N_{0}$ varies from 45 to 53 , and $N_{1}$ ranges from 36 to 37, which indicates there are a large number of new-born or damaged leaves. $R_{\text {sscan }}$ ranges from $94.59 \%$ to $86.11 \%$. $\mathrm{R}_{\text {seg } 1}$ varies from $91.43 \%$ to $87.10 \%$. $\mathrm{R}_{-}$seg 2 is reduced from $86.43 \%$ to $75.00 \%$. The values of $R_{-}$scan, $R_{-}$seg1 , and $R_{\text {seg }} 2$ indicate that occlusion affects data scanning and segmentation accuracy a lot for the occluded plants. 
Table 3. Accuracy of data scanning and individual leaf segmentation of typical leaf samples. NO. is the number of plants. $N_{0}$ is the number of leaves on the plant. $N_{1}, N_{2}$, and $N_{3}$ are the numbers of manually selected, well scanned, and automatically selected typical leaf samples. $R_{1}, R_{2}$, and $R_{3}$ are the average value of $R_{-}$scan, $R_{-}$seg1, and $R_{\_}$seg2 for each group.

\begin{tabular}{|c|c|c|c|c|c|c|c|c|c|c|c|}
\hline Groups & NO. & $N_{0}$ & $N_{1}$ & $\mathrm{~N}_{2}$ & $N_{3}$ & R_scan & R_seg1 & R_seg2 & $\mathbf{R}_{1}$ & $\mathbf{R}_{2}$ & $\mathbf{R}_{3}$ \\
\hline \multirow{3}{*}{$\begin{array}{c}1 \\
\text { (No occlusion) }\end{array}$} & 1 & 3 & 3 & 3 & 3 & $100.00 \%$ & $100.00 \%$ & $100.00 \%$ & \multirow{3}{*}{$100.00 \%$} & \multirow{3}{*}{$100.00 \%$} & \multirow{3}{*}{$100.00 \%$} \\
\hline & 2 & 6 & 6 & 6 & 6 & $100.00 \%$ & $100.00 \%$ & $100.00 \%$ & & & \\
\hline & 3 & 8 & 6 & 6 & 6 & $100.00 \%$ & $100.00 \%$ & $100.00 \%$ & & & \\
\hline \multirow{3}{*}{ (A little occlusion) } & 4 & 10 & 8 & 8 & 8 & $100.00 \%$ & $100.00 \%$ & $100.00 \%$ & \multirow{3}{*}{$100.00 \%$} & \multirow{3}{*}{$100.00 \%$} & \multirow{3}{*}{$100.00 \%$} \\
\hline & 5 & 10 & 10 & 10 & 10 & $100.00 \%$ & $100.00 \%$ & $100.00 \%$ & & & \\
\hline & 6 & 11 & 11 & 11 & 11 & $100.00 \%$ & $100.00 \%$ & $100.00 \%$ & & & \\
\hline \multirow{3}{*}{$\begin{array}{c}3 \\
\text { (Medium occlusion) }\end{array}$} & 7 & 26 & 24 & 23 & 22 & $95.83 \%$ & $95.65 \%$ & $91.67 \%$ & \multirow{3}{*}{$96.20 \%$} & \multirow{3}{*}{$94.94 \%$} & \multirow{3}{*}{$91.33 \%$} \\
\hline & 8 & 32 & 25 & 24 & 23 & $96.00 \%$ & $95.83 \%$ & $92.00 \%$ & & & \\
\hline & 9 & 34 & 31 & 30 & 28 & $96.77 \%$ & $93.33 \%$ & $90.32 \%$ & & & \\
\hline \multirow{3}{*}{$\begin{array}{c}4 \\
\text { (Heavy occlusion) }\end{array}$} & 10 & 45 & 37 & 35 & 32 & $94.59 \%$ & $91.43 \%$ & $86.49 \%$ & \multirow{3}{*}{$89.96 \%$} & \multirow{3}{*}{$88.80 \%$} & \multirow{3}{*}{$79.95 \%$} \\
\hline & 11 & 50 & 37 & 33 & 29 & $89.19 \%$ & $87.88 \%$ & $78.38 \%$ & & & \\
\hline & 12 & 53 & 36 & 31 & 27 & $86.11 \%$ & $87.10 \%$ & $75.00 \%$ & & & \\
\hline all & $1-12$ & 288 & 234 & 220 & 205 & $94.02 \%$ & $93.18 \%$ & $87.61 \%$ & - & - & - \\
\hline
\end{tabular}

In general, for plants from the first group to the fourth group, the average data scanning accuracy $R_{1}$ is $100-89.96 \%$ and segmentation accuracy $R_{2}$ and $R_{3}$ are $100-88.8 \%$ and $100-79.95 \%$. $R_{1}, R_{2}$, and $R_{3}$ decrease with the increase of canopy occlusion. The difference between $R_{2}$ and $R_{3}$ shows that the main factor that affects the segmentation results is the incomplete scanning data, which indicates the occlusion is the main factor that affects the segmentation accuracy. Generally, for all plants with different canopy occlusions, $R_{-}$scan, $R_{\text {_seg1 }}$, and $R_{\_}$seg2 are $94.02 \%, 93.18 \%$, and $87.61 \%$, respectively.

Figure 6 shows the segmentation results of typical leaf samples of different canopyoccluded plants. Figure $6 a-c$ show that the proposed method can successfully segment typical leaf samples from plants with no occlusion, a little occlusion, and medium occlusion. Small new-born leaves that are not suitable for morphological trait measurement, such as the leaves in the red boxes in Figure 6, are automatically removed. Figure 6d illustrates that most of the typical leaf samples can be successfully extracted from heavily occluded plants. Leaves with incomplete scanning data (the leaves in the yellow boxes in Figure 6) are automatically removed. The number of typical leaves that are not successfully extracted increases as the number of lost scanning data increases.

Table 3 and Figure 6 both show that the scanning and segmentation accuracy decreases with the increase of canopy occlusion. The increase of leaves and the aggravation of occlusion make the structure of the plant more complex, which increases the difficulty of data scanning and individual leaf segmentation. Specifically, on the one hand, the complex structure of the occluded plants directly affects the segmentation results; on the other hand, occlusion will affect the integrity of the scanning data and indirectly affect the segmentation accuracy. 

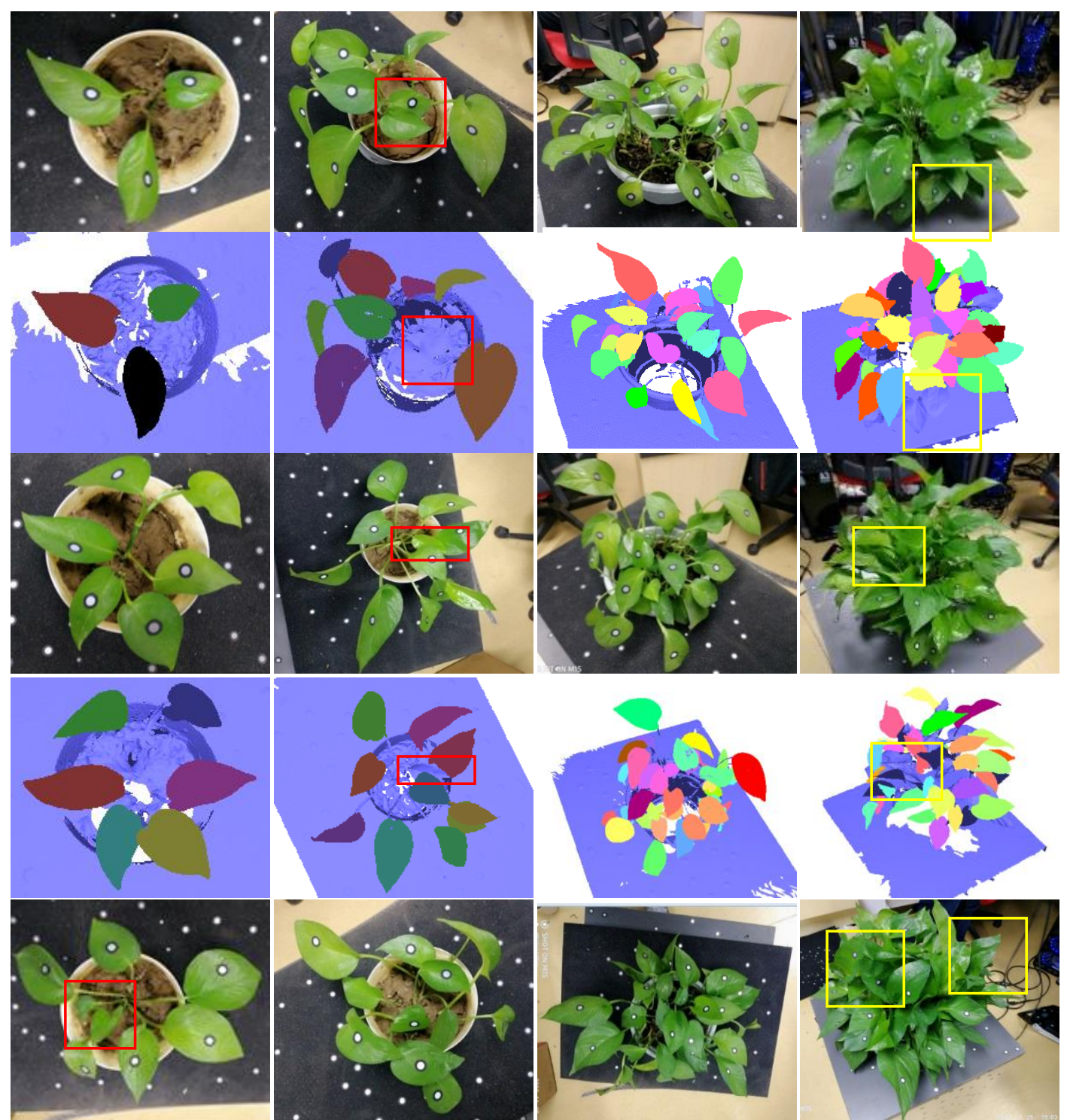

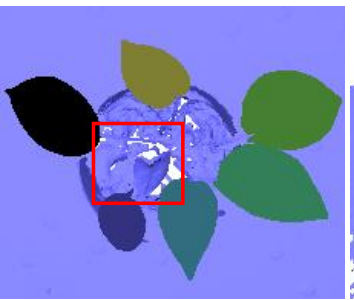

(a)

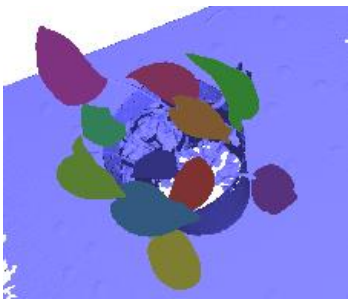

(b)

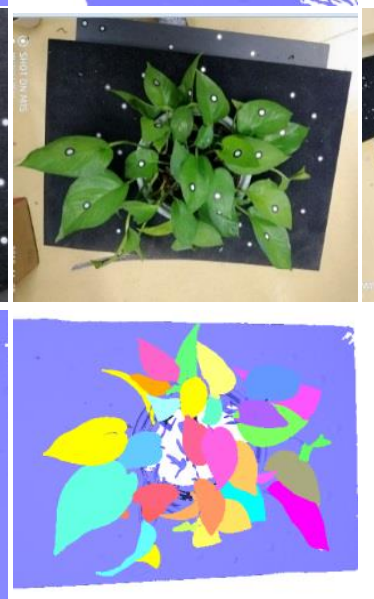

(c)

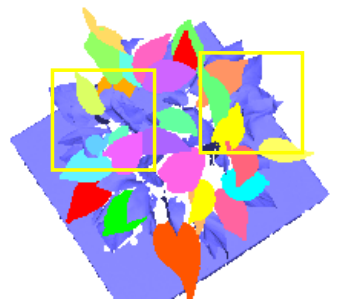

(d)

Figure 6. The automatic segmentation results of typical leaf samples of different canopy-occluded plants. (a) No canopy occlusion (group 1); (b) a little canopy occlusion (group 2); (c) medium canopy occlusion (group 3); (d) heavy canopy occlusion (group 4). The red boxes mark the new-born leaves, and the yellow boxes mark the leaves with incomplete scanning data.

\subsection{Results of Morphological Trait Estimation}

\subsubsection{Results of Scale-related Morphological Traits}

Scale-related traits contain leaf area, perimeter, length, and width in this paper. The comparison between the automatically estimated and the manually measured scale-related traits are performed. 
Figure 7 illustrates the regression analysis between automatic and manual measurements of scale-related traits of plants with no occlusion (plants in group 1). The average values of leaf area, perimeter, length, and width are $2017.50 \mathrm{~mm}^{2}, 180.65 \mathrm{~mm}, 65.03 \mathrm{~mm}$, and $41.13 \mathrm{~mm}$, respectively. Regression analyses show strong correlations $(\mathrm{EF}=0.9997$, $\mathrm{EF}=0.9861, \mathrm{EF}=0.9132$, and $\mathrm{EF}=0.9156$ for the area, perimeter, length, and width, respectively). Nevertheless, the RMSE values of the area, perimeter, length, and width are $13.59 \mathrm{~mm}^{2}, 4.73 \mathrm{~mm}, 4.09 \mathrm{~mm}$, and $2.78 \mathrm{~mm}$, respectively. The MAPE values of area, perimeter, length, and width are $0.60 \%, 2.16 \%, 5.29 \%$, and $5.79 \%$, respectively. Regression analyses, RMSE, and MAPE results show that the deviation between automatic estimation and manual measurement is very small.
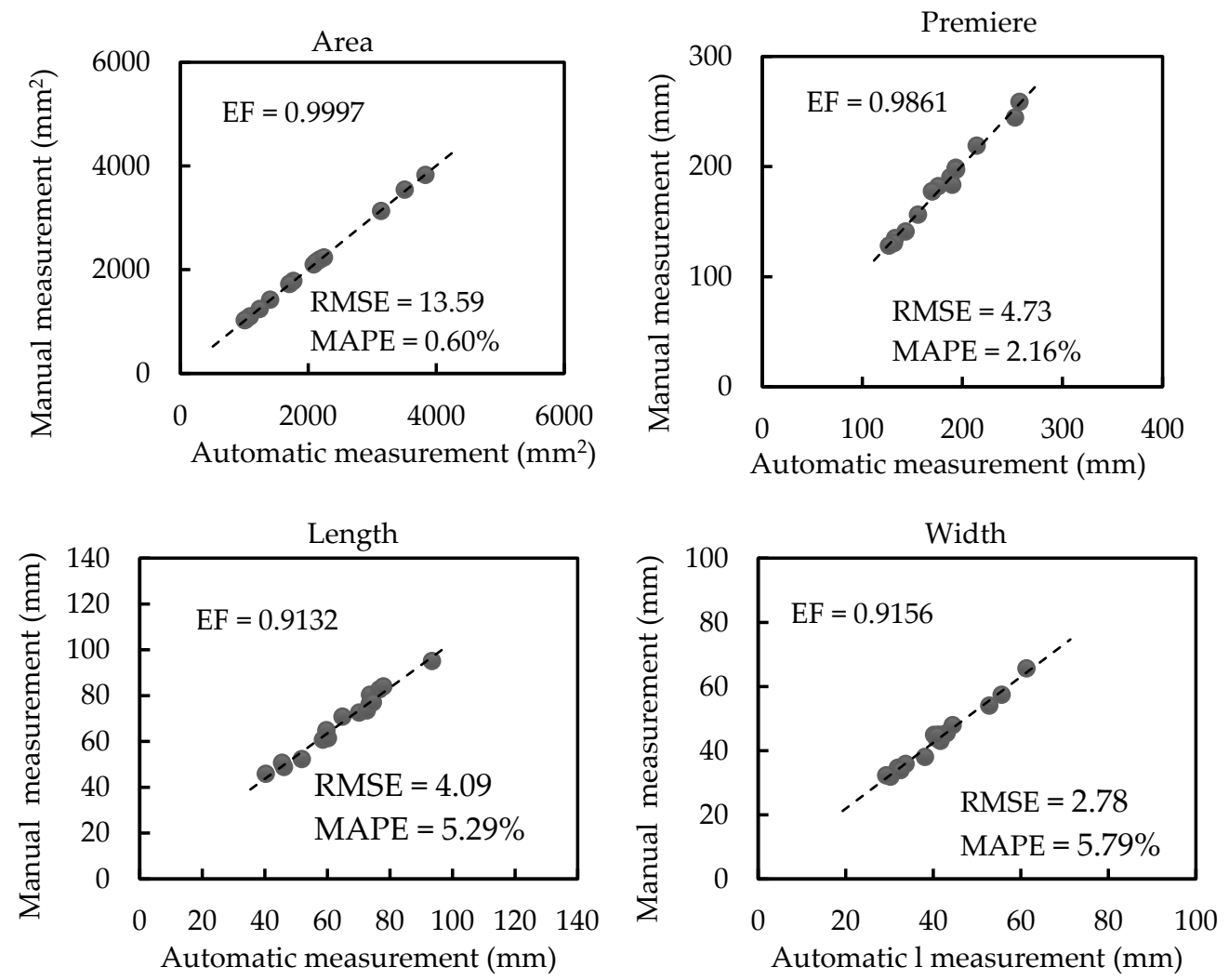

Figure 7. Regression analyses between automatic and manual measurements of scale-related traits of plants with no occlusion (plants in group 1).

Figure 8 shows regression analyses between automatic and manual measurements of scale-related traits of plants with a little occlusion (plants in group 2). As shown in Figure 8, the average leaf area, perimeter, length, and width are $2260.46 \mathrm{~mm}^{2}, 189.2 \mathrm{~mm}, 68.3 \mathrm{~mm}$, and $43.34 \mathrm{~mm}$, respectively. The automatically calculated values are highly correlated with the manually measured values based on the proposed method $(\mathrm{EF}=0.9997, \mathrm{EF}=0.9838$, $\mathrm{EF}=0.9076$, and $\mathrm{EF}=0.9080$ for the area, perimeter, length, and width, respectively). Additionally, the RMSE values of the area, perimeter, length, and width are $16.97 \mathrm{~mm}^{2}$, $5.36 \mathrm{~mm}, 5.14 \mathrm{~mm}$, and $2.88 \mathrm{~mm}$, respectively. The MAPE values of the area, perimeter, length, and width are $0.66 \%, 2.34 \%, 6.58 \%$, and $5.96 \%$, respectively. The regression analyses and the results of RMSE and MAPE exhibit little deviation of automatic measurement values from manual measurement values for plants with a little occlusion. 

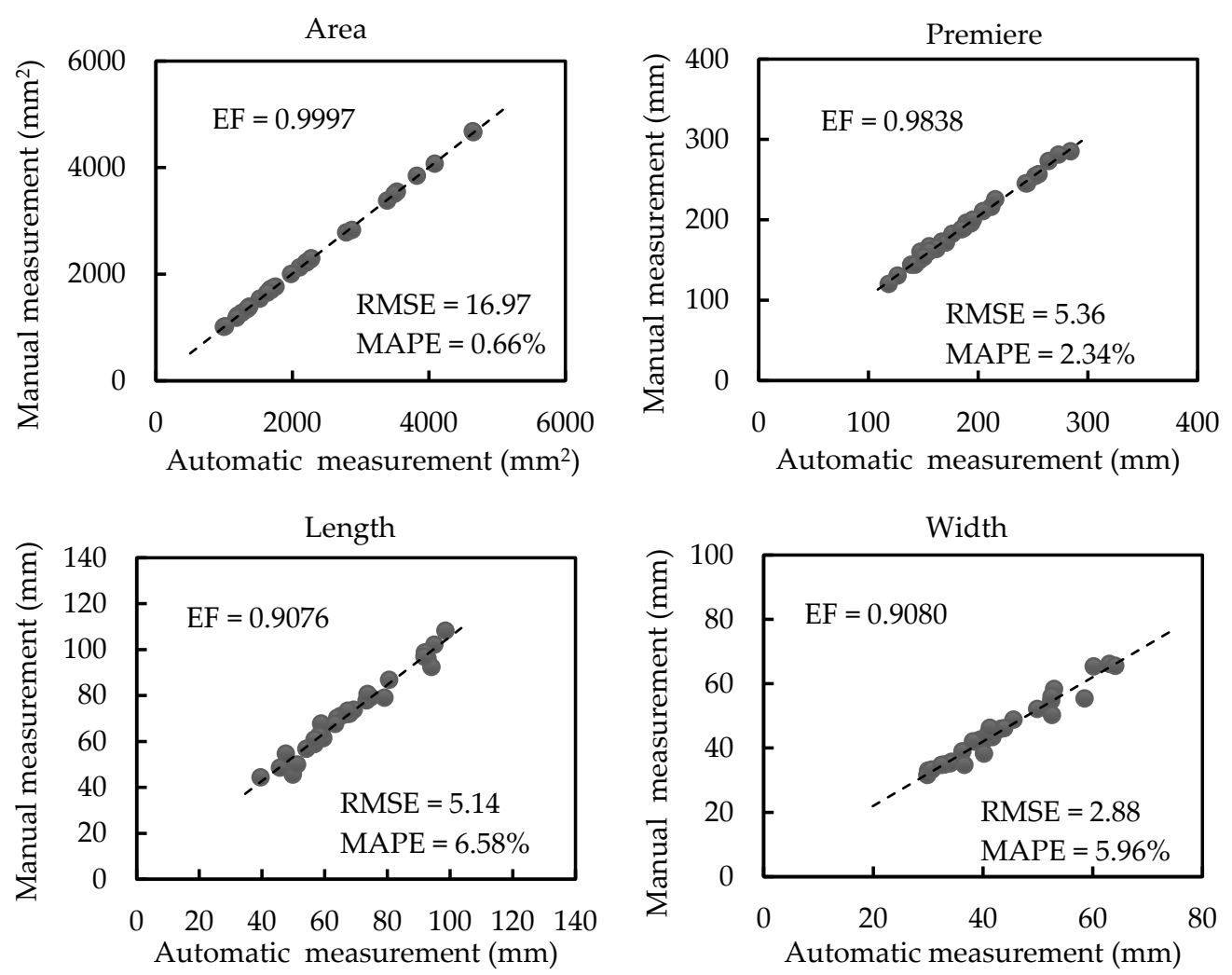

Figure 8. Regression analyses between automatic and manual measurements of scale-related traits of plants with a little occlusion (plants in group 2).

Figure 9 displays regression analyses between automatic and manual measurements of scale-related traits of plants with medium occlusion (plants in group 3). The leaf area, perimeter, length, and width have average values of $2270.66 \mathrm{~mm}^{2}, 190.35 \mathrm{~mm}, 69.33 \mathrm{~mm}$, and $44.71 \mathrm{~mm}$, respectively. EF for the area, perimeter, length, and width, are $0.9993,0.9784$, 0.8787 , and 0.8857 , respectively. This indicates the automatically calculated values are highly correlated with the manually measured values. Furthermore, the RMSE values of the area, perimeter, length, and width are $26.13 \mathrm{~mm}^{2}, 6.45 \mathrm{~mm}, 5.73 \mathrm{~mm}$, and $3.32 \mathrm{~mm}$, respectively, and the MAPE values of the area, perimeter, length, and width are $0.68 \%$, $2.66 \%, 6.74 \%$, and $6.03 \%$, respectively. The regression analyses and the results of RMSE and MAPE exhibit a small deviation of automatic measurement values from manual measurement values for plants with medium occlusion.

Figure 10 exhibits regression analyses between automatic and manual measurements of scale-related traits of plants with heavy occlusion (plants in group 4). Meanwhile, values of RMSE and MAPE are exhibited. The average leaf area, perimeter, length, and width have values of $3845.10 \mathrm{~mm}^{2}, 252.48 \mathrm{~mm}, 89.75 \mathrm{~mm}$, and $59.06 \mathrm{~mm}$, respectively. EF for area, perimeter, length, and width, are $0.9981,0.9600,0.7536$, and 0.7502 , respectively. This illustrates the automatic measurement values have strong explicit correlations with the manual measurement values. Moreover, the RMSE values of the area, perimeter, length, and width are $48.13 \mathrm{~mm}^{2}, 8.21 \mathrm{~mm}, 7.37 \mathrm{~mm}$, and $4.41 \mathrm{~mm}$, respectively, and the MAPE values of the area, perimeter, length, and width are $0.77 \%, 2.87 \%, 7.03 \%$, and $6.85 \%$, respectively. The regression analyses and the results of RMSE and MAPE indicate a small deviation of automatic measurement values from manual measurement values for plants with heavy occlusion. 

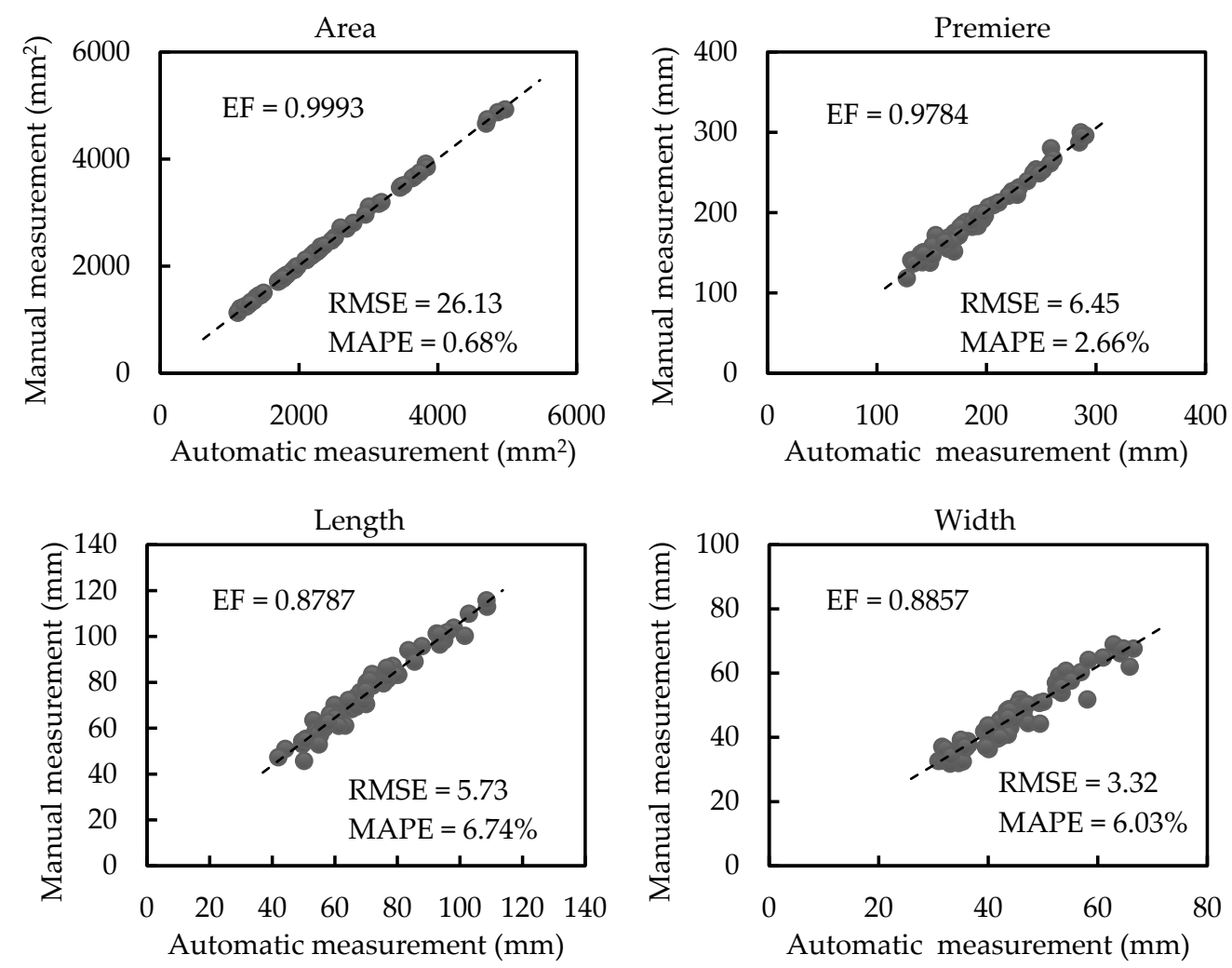

Figure 9. Regression analyses between automatic and manual measurements of scale-related traits of plants with medium occlusion (plants in group 3).
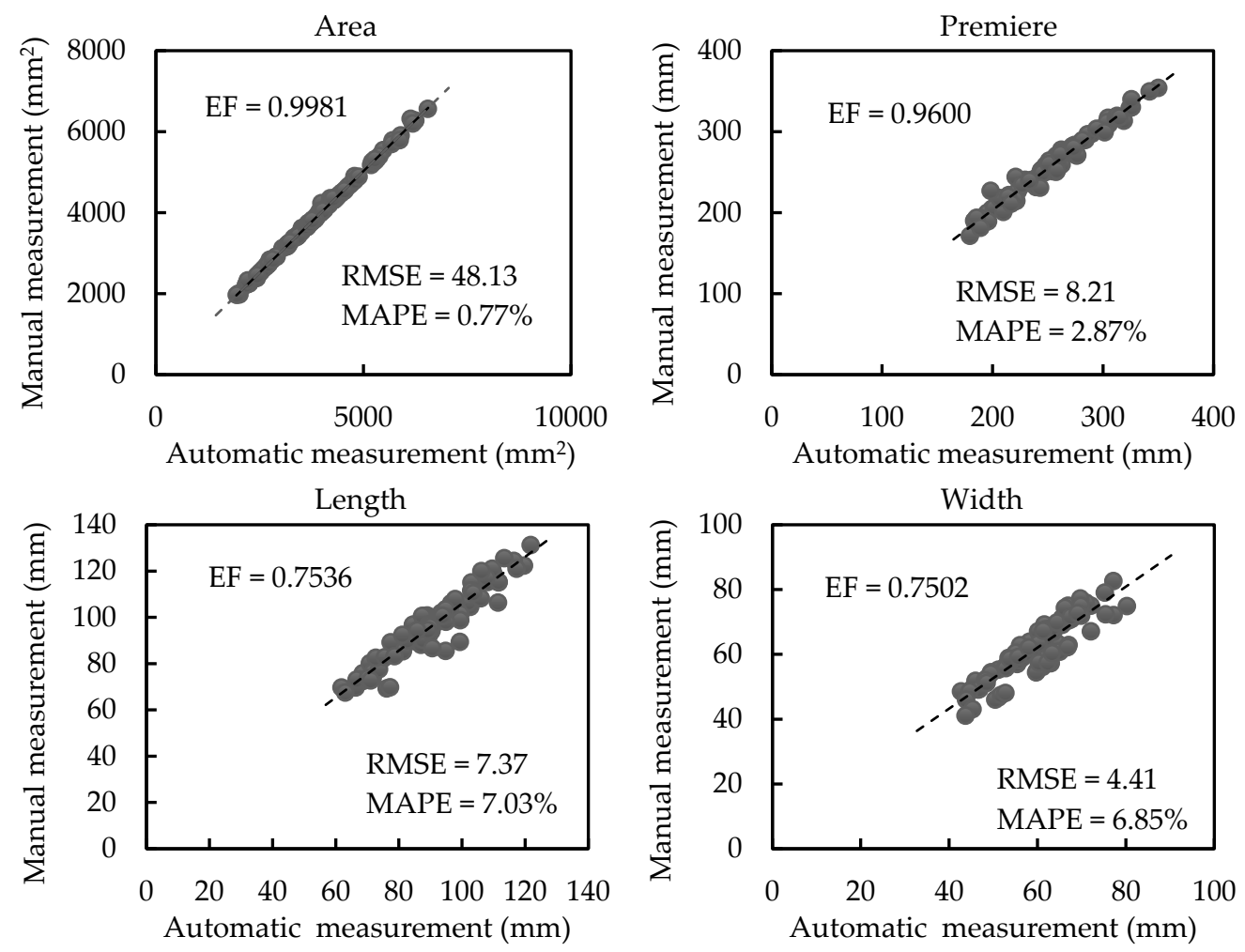

Figure 10. Regression analyses between automatic and manual measurements of scale-related traits of plants with heavy occlusion (plants in group 4). 
Figure 11 exhibits regression analyses between automatic and manual measurements of scale-related traits of plants with different occlusion (plants in all groups). The average values of leaf area, perimeter, length, and width have values of $2946.10 \mathrm{~mm}^{2}, 216.92 \mathrm{~mm}$, $77.88 \mathrm{~mm}$, and $50.59 \mathrm{~mm}$, respectively. The automatically calculated values are highly correlated with the manually measured values based on the proposed method $(\mathrm{EF}=0.9992$, $\mathrm{EF}=0.9827, \mathrm{EF}=0.8919$, and $\mathrm{EF}=0.9039$ for the area, perimeter, length, and width, respectively). Additionally, the RMSE values of the area, perimeter, length, and width are $14.12 \mathrm{~mm}^{2}, 4.11 \mathrm{~mm}, 3.42 \mathrm{~mm}$, and $1.98 \mathrm{~mm}$, respectively. The MAPE values of the area, perimeter, length, and width are $0.72 \%, 2.69 \%, 6.83 \%$, and $7.16 \%$, respectively. All the regression analyses and the results of RMSE and MAPE comparing manual measurement traits versus automatic measurement traits for plants with different occlusions show that the accuracy of the proposed morphological trait estimation method is competitive and effective.
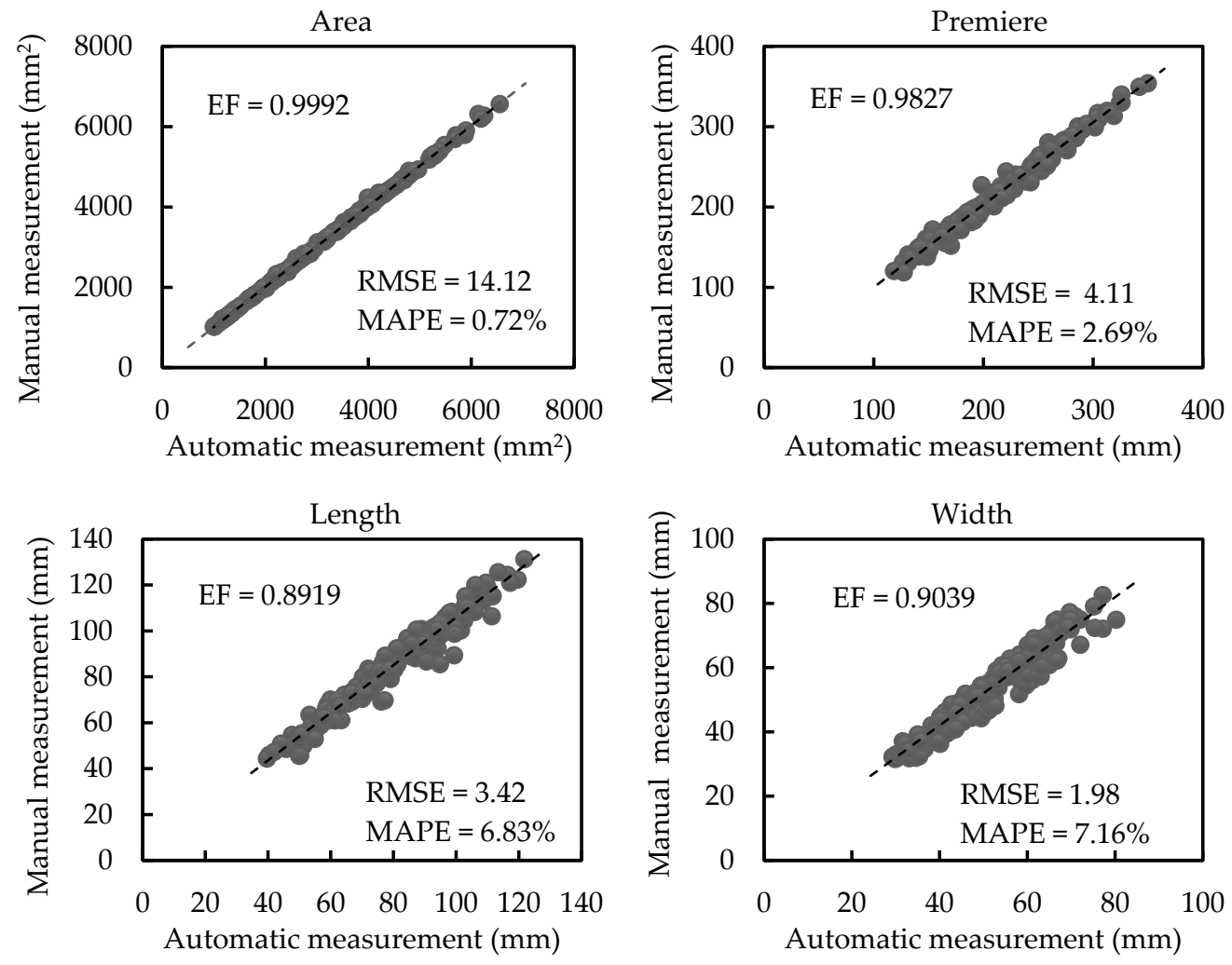

Figure 11. Regression analyses between automatic and manual measurements of scale-related traits of plants with different occlusions (plants in all groups).

\subsubsection{Results of Scale-invariant Morphological Traits}

Six scale-invariant traits are estimated. The average scale-invariant traits $\mathrm{X}_{11}, \mathrm{X}_{12}, \mathrm{X}_{13}$, $X_{14}, X_{15}$, and $X_{16}$ are $12.93,36.14,55.56,2.79,4.30$, and 1.55 , respectively. Table 4 shows the values of $\mathrm{EF}$ of regression analyses between the automatic and manual measurement of scale-invariant traits of plants with different occlusions. For plants from the first group to the fourth group, $\mathrm{EF}$ for the scale-invariant traits $\mathrm{X}_{11}, \mathrm{X}_{12}, \mathrm{X}_{13}, \mathrm{X}_{14}, \mathrm{X}_{15}$, and $\mathrm{X}_{16}$ have values of $0.9883-0.9579,0.9202-0.8461,0.9386-0.8276,0.8728-0.7775,0.8312-0.6891$, and $0.8268-0.6895$, respectively. 
Table 4. The values of EF of regression analyses between the automatic and manual measurement of scale-invariant traits for plants with different occlusions.

\begin{tabular}{ccccccc}
\hline Groups & $\mathbf{X}_{\mathbf{1 1}}$ & $\mathbf{X}_{\mathbf{1 2}}$ & $\mathbf{X}_{\mathbf{1 3}}$ & $\mathbf{X}_{\mathbf{1 4}}$ & $\mathbf{X}_{\mathbf{1 5}}$ & $\mathbf{X}_{\mathbf{1 6}}$ \\
\hline 1 & 0.9857 & 0.9202 & 0.9102 & 0.8728 & 0.8312 & 0.8268 \\
2 & 0.9883 & 0.9130 & 0.9386 & 0.8513 & 0.8147 & 0.7594 \\
3 & 0.9692 & 0.8507 & 0.9017 & 0.8472 & 0.7449 & 0.7322 \\
4 & 0.9579 & 0.8461 & 0.8276 & 0.7775 & 0.6891 & 0.6895 \\
All & 0.9811 & 0.8908 & 0.9162 & 0.8509 & 0.7838 & 0.7434 \\
\hline
\end{tabular}

The measurement accuracy of scale-invariant traits of plants with different occlusions is listed in Table 5. For plants from the first group to the fourth group, the results of RMSE have values of $0.27-0.44,1.83-3.19,2.83-4.47,0.17-0.23,0.23-0.34$, and $0.07-0.14$ for traits $X_{11}, X_{12}, X_{13}, X_{14}, X_{15}$, and $X_{16}$, respectively. The results of MAPE have values of $1.95-2.65 \%, 5.30-7.08 \%, 5.76-6.78 \%, 4.83-7.07 \%, 4.56-7.04 \%$, and 3.38-6.19\% for traits $X_{11}$, $\mathrm{X}_{12}, \mathrm{X}_{13}, \mathrm{X}_{14}, \mathrm{X}_{15}$, and $\mathrm{X}_{16}$, respectively. The results of RMSE and MAPE increase with the increase of occlusion degree, which indicates that the measurement accuracy decreases with the increase of occlusion degree. The differences of RMSE and MAPE increase overall from the first group to the fourth group.

Table 5. The measurement accuracy of scale-invariant traits for plants with different occlusions.

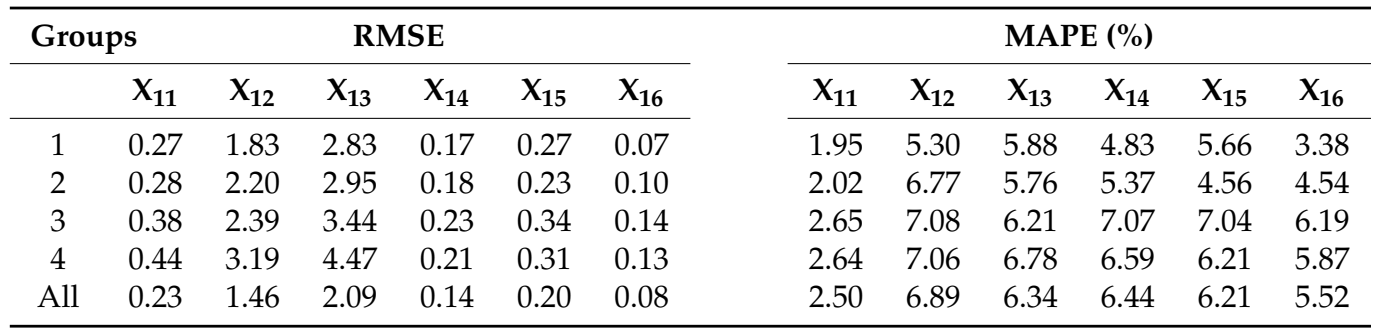

For all plants, the automatically calculated values are correlated with the manually measured values based on the proposed method $(\mathrm{EF}=0.9811, \mathrm{EF}=0.8908, \mathrm{EF}=0.9162$, $\mathrm{EF}=0.8509, \mathrm{EF}=0.7838$, and $\mathrm{EF}=0.7434$ for traits $\mathrm{X}_{11}, \mathrm{X}_{12}, \mathrm{X}_{13}, \mathrm{X}_{14}, \mathrm{X}_{15}$, and $\mathrm{X}_{16}$, respectively). In addition, the RMSE values of traits $X_{11}, X_{12}, X_{13}, X_{14}, X_{15}$, and $X_{16}$ are $0.23,1.46$, $2.09,0.14,0.20$, and 0.08 , respectively, and the MAPE values of the corresponding traits are $2.50 \%, 6.89 \%, 6.34 \%, 6.44 \%, 6.21 \%$, and $5.52 \%$, respectively.

\subsection{Time Cost}

Table 6 lists the time cost of the experiments. For all the experiments, the data scanning time ranges from 120 to 250 seconds. The output time of a 3D model of a plant is between 5.68 and 6.24 seconds. The data processing time including segmentation and trait estimation varies from 4.02 to 5.13 seconds. Data scanning occupies most of the time. In general, the average measurement time of one plant is 196.37 seconds (186.08 seconds for data scanning, 5.95 seconds for 3D model output, and 4.36 seconds for automatic selection of typical leaf samples and morphological trait estimation). 
Table 6. Time cost (second). T_scan: time of data scanning. T_mesh: time of 3D model output. T_p: time of data processing.

\begin{tabular}{cccccccccc}
\hline Plants & Points & T_scan & T_mesh & T_p & Plants & Points & T_scan & T_mesh & T_p \\
\hline 1 & 203,343 & 120 & 5.68 & 4.02 & 7 & 434,177 & 185 & 6.08 & 4.25 \\
2 & 279,350 & 130 & 5.71 & 4.02 & 8 & 400,144 & 191 & 6.00 & 4.27 \\
3 & 304,232 & 140 & 5.81 & 4.02 & 9 & 420,915 & 199 & 6.05 & 4.29 \\
4 & 364,758 & 160 & 5.89 & 4.08 & 10 & 449,642 & 265 & 6.12 & 5.13 \\
5 & 364,299 & 170 & 5.88 & 4.09 & 11 & 456,619 & 247 & 6.13 & 5.04 \\
6 & 359,396 & 176 & 5.81 & 4.09 & 12 & 493,968 & 250 & 6.24 & 5.06 \\
\hline
\end{tabular}

\section{Discussion}

\subsection{Measurements on Different Canopy-Occluded Plants}

In this paper, a novel method based on a handheld laser scanner to automatically measure the morphological traits of typical leaf samples of different canopy-occluded live plants was proposed. Plants with different canopy occlusions were tested. The experimental results show that the measurement accuracy of the proposed method for automatically estimating morphological traits of leaves is competitive. It should be noted that the measurement accuracy is different when measuring plants with different canopy occlusions.

The presented results of scanning (Table 3) show that the scanning accuracy is decreased with the increase of canopy occlusion of plants. The plants with no and a little canopy occlusion can be well scanned (both with scanning accuracy $100 \%$ ), and the plants with medium and heavy canopy occlusion can lose some data (with scanning accuracy $96.20 \%$ and $89.96 \%$, respectively). This is because plants with heavier canopy occlusion have more attached and overlapped leaves, which makes data scanning more difficult. This problem is common in data acquisition based on non-penetration devices $[27,28,33]$.

The presented results of segmentation (Table 3 and Figure 6) show that the segmentation accuracy is decreased with the increase of canopy occlusion of plants. $\mathrm{R}_{2}$ varies from $100 \%$ to $88.8 \% \mathrm{R}_{3}$ has values from $100 \%$ to $79.95 \%$. There are two reasons. On one hand, the increase of canopy occlusion makes the lack of scanning data increase, which decreases the segmentation accuracy indirectly. On the other hand, the increase of canopy occlusion makes the complexity of plant geometric structure increase, which increases the difficulty of the segmentation algorithm directly. This is the reason that the experiment subjects in most contemporary research $([18,19,23])$ are plants with several leaves at the early growth stage when measuring leaf morphological traits. Additionally, the difference between $R_{2}$ and $R_{3}$ shows that the main factor that affects the segmentation results is the incomplete scanning data, which indicates the occlusion is the main factor that affects the segmentation accuracy.

The presented results of morphological trait estimation (Figures 7-10 and Tables 4 and 5) show that the trait estimation accuracy is decreased with the increase of canopy occlusion of the plants. There are two main reasons. On one hand, the occlusion decreases the segmentation accuracy, which influences the trait estimation indirectly. On the other hand, the occlusion leads to less growth space, which influences the geometrical shape of leaves and directly affects the trait calculation algorithms. Regarding the regression analyses between automatic and manual measurements of morphological traits, the values of EF are decreased with the increase of plant canopy occlusion. Specifically, the values of $\mathrm{EF}$ for the area, perimeter, length, width, $\mathrm{X}_{11}, \mathrm{X}_{12}, \mathrm{X}_{13}, \mathrm{X}_{14}, \mathrm{X}_{15}$, and $\mathrm{X}_{16}$ vary from $0.9997,0.9861,0.9132,0.9156,0.9883,0.9202,0.9386,0.8728,0.8312$, and 0.8268 to 0.9981 , $0.9600,0.7536,0.7502,0.9579,0.8461,0.8276,0.7775,0.6891$, and 0.6895 , respectively. The morphological trait measurement of plants with no canopy occlusion has high values of EF, and the plants with heavy canopy occlusion have low values. This illustrates the measurement accuracy of plants with no canopy occlusion is higher than that of plants with heavy occlusion. The values of $\mathrm{EF}$ in regression analyses are all positive, which illustrates the automatically calculated values are correlated with the manually measured values. Figures 7-10 also illustrate the automatic measurements are underestimated compared to 
manual measurements. This is because it is easy to lose some data in data scanning and leaf segmentation. Regarding the RMSE and MAPE between the automatic and manual measurements of morphological traits, the values of RMSE and MAPE are increased with the increase of plant canopy occlusion. Specifically, the values of RMSE of the area, perimeter, length, width, $\mathrm{X}_{11}, \mathrm{X}_{12}, \mathrm{X}_{13}, \mathrm{X}_{14}, \mathrm{X}_{15}$, and $\mathrm{X}_{16}$ vary from $13.59 \mathrm{~mm}^{2}, 4.73 \mathrm{~mm}$, $4.09 \mathrm{~mm}$, and $2.78 \mathrm{~mm}, 0.27,1.83,2.83,0.17,0.23$, and 0.07 to $48.13 \mathrm{~mm}^{2}, 8.21 \mathrm{~mm}, 7.37 \mathrm{~mm}$, $4.41 \mathrm{~mm}, 0.44,3.19,4.47,0.21,0.34$, and 0.14 , respectively. The values of MAPE of the area, perimeter, length, width, $\mathrm{X}_{11}, \mathrm{X}_{12}, \mathrm{X}_{13}, \mathrm{X}_{14}, \mathrm{X}_{15}$, and $\mathrm{X}_{16}$ vary from $0.60 \%, 2.16 \%$, $5.29 \%, 5.79 \%, 1.95 \%, 5.30 \%, 5.76 \%, 4.83 \%, 4.56 \%$, and $3.38 \%$ to $0.77 \%, 2.87 \%, 7.03 \%, 6.85 \%$, $2.65 \%, 7.08 \%, 6.78 \%, 7.07 \%, 7.04 \%$, and $6.19 \%$, respectively. It can be found out that the morphological trait measurement of plants with no canopy occlusion has low values of RMSE and MAPE and the plants with heavy canopy occlusion have high values. The values of RMSE exhibit that the automatic trait estimation accuracy can reach the military level. The values of MAPE of the area and perimeter are smaller than those of length and width in the same canopy occlusion condition. This is because calculation algorithms of length and width are based on the 3D mesh of the leaf. The values of MAPE of the scale-related traits are lower than those of scale-invariant traits. This is because that the scale-invariant traits are derived from the scale-related traits.

It should be noted that varied results between the automatically and manually measured morphological traits are usually found when leaves are attached or overlapped. The deviation becomes larger when there are more connected and overlapped parts. This is because the attached or overlapped leaves make segmentation difficult. What is worse, the leaves with a large area of connected and overlapped parts would fail to be segmented.

The presented time cost (Table 6) shows that the time cost increases with the increase of the canopy occlusion. It can be found out that the main time cost is in the data scanning. Plants with more complicated canopy occlusion require more time for data acquisition. The data processing is really fast and the difference in the time cost of different canopy-occluded plants can be ignored. The low time cost indicates the proposed method is competitive and has the potential for real-time plant measurement.

It can be found out although the measurement accuracies from plants with no canopy occlusion to heavy occlusion are reduced slightly, the overall accuracies of scanning, segmentation, and morphological trait estimation are competitive (Figure 11). The time cost of the proposed method for different canopy-occluded plants is low. The competitive measurement accuracy and low time cost of the proposed method show great potential for real-time plant measurement and high-throughput plant phenotype.

\subsection{Comparison of Related Methods}

In this paper, a multi-level region growing segmentation using two leaf shape models was proposed to perform individual leaf segmentation and morphological trait estimation. The segmentation comparison of two widely used segmentation methods and our segmentation method is presented in Figure 12. Method A is the Euclidean Clustering method in papers [24,29] and B is the Facet Region Growing method in paper [27]. It can be found out that the Euclidean Clustering method performs well in plants with no and a little canopy occlusion. Some attached leaves are failed to be segmented based on the Euclidean Clustering method when the plants have medium canopy occlusion. Large numbers of leaves are failed to be segmented based on the Euclidean Clustering method when the plants have heavy canopy occlusion. It should be noted that the segmented leaves with the Euclidean Clustering method are usually with stems (the leaves in the yellow boxes in Figure 12), which will affect the leaf measurement accuracy in the morphological trait calculation process. Regarding the Facet Region Growing method, it performs well for plants with no, a little, and medium canopy occlusion. However, some attached and overlapped leaves (the leaves in the red boxes in Figure 12) fail to be segmented using Facet Region Growing method when plants are heavily occluded. It can be seen that the number of leaves that could not be segmented by the Facet Region Growing method is 
more than ours. It should be noted that the segmentation results using both the Euclidean Clustering method and Facet Region Growing method includes leaves with incomplete scanning data and newborn leaves, which will affect the morphological trait estimation accuracy. It should be noted that the typical leaf samples that are suitable for morphological trait estimation can be automatically selected using our proposed method, while the other two segmentation cannot.

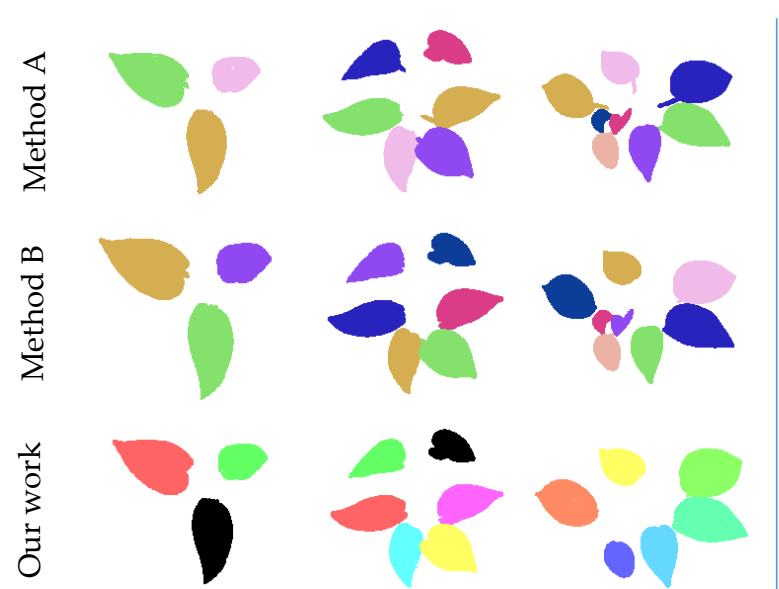

(a)

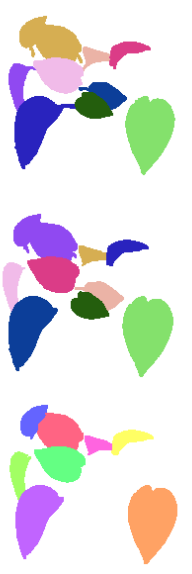

(b)

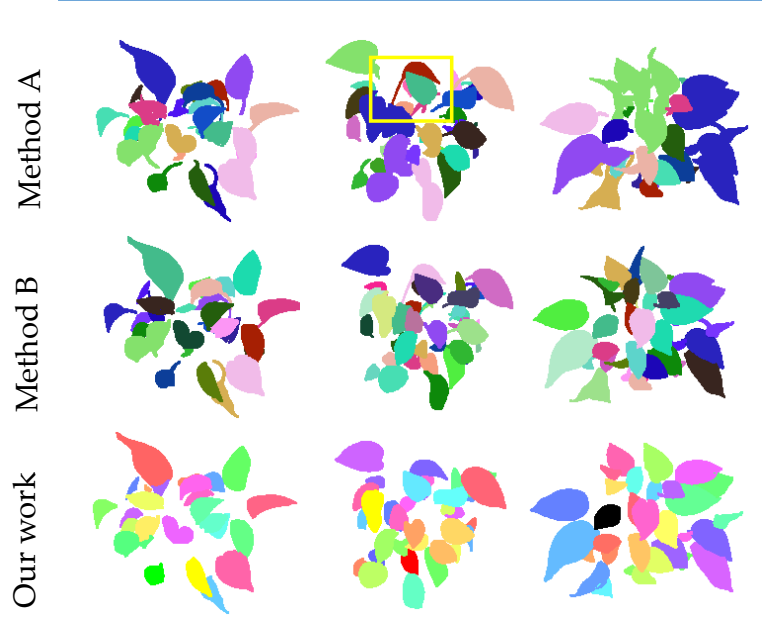

(c)
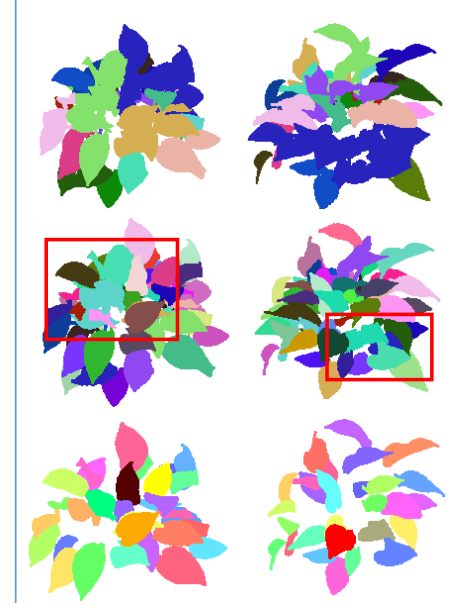

(d)

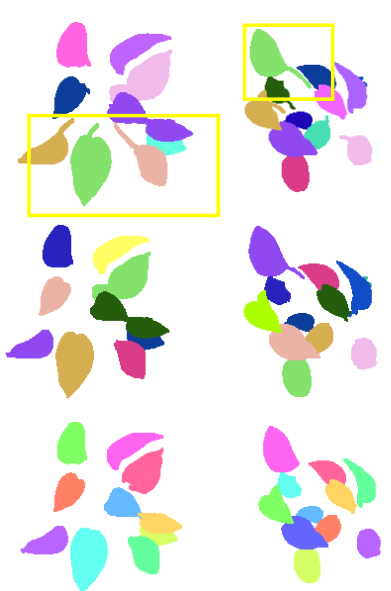

(b)

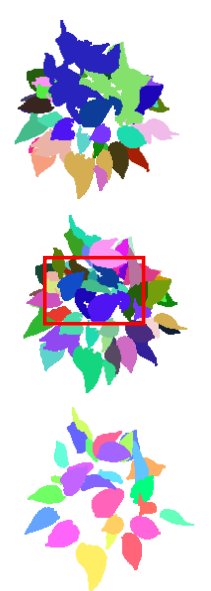

Figure 12. Segmentation results of two segmentation methods and our proposed method. Method A is the Euclidean Clustering method in papers [24,29] and B is the Facet Region Growing method in paper [27]. Plants in (a), (b), (c), and (d) are plants with no, a little, medium, and heavy canopy occlusion. The yellow boxes mark some segmented results of leaves with stems using the Euclidean Clustering method. The red boxes mark some segmented results of attached and overlapped leaves using the Facet Region Growing method.

The measurement accuracies of morphological traits using the Euclidean Clustering method, Facet Region Growing method, and our proposed method are listed in Tables 7 and 8 . The values of EF of automatically measured and the manually measured traits using the Euclidean Clustering method, the Facet Region Growing method, and our proposed are increased. The values of RMSE and MAPE using the Euclidean Clustering method, the Facet Region Growing method, and our proposed are decreased. It can be found out that the measurement accuracies using the Facet Region Growing method are higher than those employing the Euclidean Clustering method. The proposed method has higher measurement accuracies compared to the Facet Region Growing method and the Euclidean Clustering method. 
Table 7. Measurement accuracies of the scale-related morphological traits using the Euclidean Clustering method, Facet Region Growing method, and our method.

\begin{tabular}{cccccc}
\hline Methods & & Area & Perimeter & Length & Width \\
\hline Method A & EF & 0.8430 & 0.8299 & 0.7872 & 0.7501 \\
& RMSE & $54.98 \mathrm{~mm}^{2}$ & $18.56 \mathrm{~mm}$ & $11.44 \mathrm{~mm}$ & $9.66 \mathrm{~mm}$ \\
& MAPE & $5.44 \%$ & $9.44 \%$ & $16.03 \%$ & $18.57 \%$ \\
Method B & EF & 0.9354 & 0.9103 & 0.8546 & 0.8212 \\
& RMSE & $23.98 \mathrm{~mm}^{2}$ & $12.13 \mathrm{~mm}$ & $7.12 \mathrm{~mm}$ & $5.47 \mathrm{~mm}$ \\
& MAPE & $2.13 \%$ & $6.12 \%$ & $9.12 \%$ & $11.57 \%$ \\
Our work & EF & 0.9992 & 0.9827 & 0.8919 & 0.9039 \\
& RMSE & $14.12 \mathrm{~mm}^{2}$ & $4.11 \mathrm{~mm}$ & $3.42 \mathrm{~mm}$ & $1.98 \mathrm{~mm}$ \\
& MAPE & $0.72 \%$ & $2.69 \%$ & $6.83 \%$ & $7.16 \%$ \\
\hline
\end{tabular}

Table 8. Measurement accuracies of the scale-related morphological traits using the Euclidean Clustering method, Facet Region Growing method, and our method.

\begin{tabular}{cccccccc}
\hline Methods & & $\mathbf{X}_{\mathbf{1 1}}$ & $\mathbf{X}_{\mathbf{1 2}}$ & $\mathbf{X}_{\mathbf{1 3}}$ & $\mathbf{X}_{\mathbf{1 4}}$ & $\mathbf{X}_{\mathbf{1 5}}$ & $\mathbf{X}_{\mathbf{1 6}}$ \\
\hline Method A & EF & 0.8286 & 0.7812 & 0.7513 & 0.7001 & 0.6108 & 0.5603 \\
& RMSE & 0.78 & 5.67 & 7.63 & 0.45 & 0.49 & 0.17 \\
& MAPE & $5.17 \%$ & $14.39 \%$ & $12.68 \%$ & $12.99 \%$ & $12.68 \%$ & $10.34 \%$ \\
Method B & EF & 0.9102 & 0.8512 & 0.8417 & 0.8103 & 0.7029 & 0.6819 \\
& RMSE & 0.46 & 3.57 & 4.09 & 0.25 & 0.30 & 0.10 \\
& MAPE & $3.59 \%$ & $10.47 \%$ & $9.46 \%$ & $9.79 \%$ & $9.56 \%$ & $7.89 \%$ \\
Our work & EF & 0.9811 & 0.8908 & 0.9162 & 0.8509 & 0.7838 & 0.7434 \\
& RMSE & 0.23 & 1.46 & 2.09 & 0.14 & 0.20 & 0.08 \\
& MAPE & $2.50 \%$ & $6.89 \%$ & $6.34 \%$ & $6.44 \%$ & $6.21 \%$ & $5.52 \%$ \\
\hline
\end{tabular}

\subsection{Advantages, Limitations, Improvements, and Future Works}

In this paper, a novel method for automatic measurement of the morphological traits of typical leaf samples of different canopy-occluded live plants using a handheld laser scanner is proposed. Our method has four advantages. First of all, the 3D mesh model of the plant obtained by ZGScan 717 has higher precision and richer details compared to those reconstructed by the SFM method [24], time-of-flight (ToF) method [22], binocular stereo vision method [27], LIDAR method [34], and FastSCAN hand-held scanner [35]. The 3D mesh model is reconstructed in real-time during data scanning and can be output directly, thus saving data processing time for 3D reconstruction in most contemporary research. Second, typical leaf samples can be automatically selected, and the accuracies of segmentation and trait estimation are competitive compared to related works [27,29,33]. A multi-level region growing segmentation method using two leaf shape models is proposed. The two main segmentation parameters, smooth and curvature, are adaptive to different input data at different segmentation scales. The two shape models, one using scale-related traits and the other using scale-invariant traits, are dynamically established based on the different segmentation results at different scales. The proposed segmentation method performs better compared with the Euclidean Clustering method in papers [24,29] and the Facet Region Growing method in paper [27]. Third, the time cost of our proposed method, especially the data processing time, is really low. Finally, compared with most of the literature $[18,19]$, which only focuses on the research of non-occluded plants at the early growth stage, the method proposed in this paper can be applied to different canopy-occluded plants.

It should be noted that the segmentation method and trait estimation algorithm are based on the 3D mesh models. It has little to do with the acquisition method of the 3D model. Hence, the proposed method in this paper is universal no matter what instrument is used for obtaining 3D mesh models of plants.

However, there are still some limitations in our research. For the process of data scanning, it is necessary to stick the mark points on and around the plant leaves in advance. 
It should be noted that the proposed method is suitable for plants with a similar leaf shape to Epipremnum aureum, such as eucalyptus, cinnamon, and gardenia. Specifically, the leaves should be broad and elliptical. Leaves with long and banding shapes, such as rice, corn, and wheat, may not be suitable. This is because their leaves are usually soft and curved, which makes the length and width calculation algorithms differ from the elliptical ones.

Thus, the improvement and future work will focus on two problems, namely trait estimation algorithm improvement and segmentation improvement.

\section{Conclusions}

This paper presents a novel method based on a hand-held 3D laser scanner to automatically extract typical leaf samples and estimate their morphological traits from live plants with different canopy occlusions. First, the 3D mesh model of the plant that is reconstructed in real-time during data scanning by a hand-held 3D laser scanner (ZGScan 717) is obtained directly. The plant model has high precision (maximum accuracy of $0.03 \mathrm{~mm}$ ) and rich details of plant architecture. Then a multi-level region growing segmentation method using two leaf shape models is conducted to automatically extract typical leaf samples and estimate their morphological traits. Particularly, the two main segmentation parameters, smooth and curvature, are adaptive to different plants at different segmentation scales, and the shape model dynamically updates based on the different segmentation results at different scales using the principal component analysis method. Four scale-related traits and six corresponding scale-invariant traits are automatically estimated. Four groups of different canopy-occluded plants are tested. Experiments show that for plants with different canopy occlusions, $94.02 \%$ of typical leaf samples can be scanned well and $87.61 \%$ of typical leaf samples can be automatically extracted. The automatically measured morphological traits are correlated with the manually measured values $(\mathrm{EF}=0.9992, \mathrm{EF}=0.9827, \mathrm{EF}=0.8919$, $\mathrm{EF}=0.9039, \mathrm{EF}=0.9811, \mathrm{EF}=0.8908, \mathrm{EF}=0.9162, \mathrm{EF}=0.8509, \mathrm{EF}=0.7838$, and $\mathrm{EF}=0.7434$ for the area, perimeter, length, width, area perimeter ratio, area length ratio, area width ratio, perimeter length ratio, perimeter width ratio, and aspect ratio, respectively). In addition, the RMSE values of those 10 morphological traits are $14.12 \mathrm{~mm}^{2}, 4.11 \mathrm{~mm}, 3.42 \mathrm{~mm}$, $1.98 \mathrm{~mm}, 0.23,1.46,2.09,0.14,0.20$, and 0.08 , respectively, and the corresponding MAPE values are $0.72 \%, 2.69 \%, 6.83 \%, 7.16 \%, 2.50 \%, 6.89 \%, 6.34 \%, 6.44 \%, 6.21 \%$, and $5.52 \%$, respectively. The average time of one plant measurement is 196.37 seconds (186.08 seconds for data scanning, 5.95 seconds for 3D model output, and 4.36 seconds for data processing).

The successful application of the proposed method on four different canopy-occluded plants shows the robustness of the proposed method in plant measurement. Therefore, it can be concluded that our method can automatically select typical leaf samples of live plants with different canopy occlusions and output 10 leaf morphological traits with high measurement accuracy and low time cost. The proposed method demonstrates the ability of rapid batch processing of data, which is potential for real-time plant measurement and high-throughput plant phenotype.

Since occlusion is the main factor affecting measurement accuracy, we are interested in exploring more effective segmentation methods, such as adding color information to improve the segmentation accuracy. We also plan to integrate the proposed method into a hand-held scanner system to achieve real-time plant measurement.

Author Contributions: Conceptualization, methodology, and investigation: X.H. and S.Z.; data acquisition and experiment: X.H.; writing-original draft preparation: X.H.; writing-review and editing: L.G.; supervision: S.Z.; funding acquisition: S.Z. All authors have read and agreed to the published version of the manuscript.

Funding: This work was supported by the Natural Sciences Foundation of China $(41671452,41701532)$.

Conflicts of Interest: The authors declare no conflict of interest. 


\section{References}

1. Apelt, F.; Breuer, D.; Nikoloski, Z.; Stitt, M.; Kragler, F. Phytotyping(4D): A light-field imaging system for non-invasive and accurate monitoring of spatio-temporal plant growth. Plant J. 2015, 82, 693-706. [CrossRef]

2. Clark, B.; Bullock, S. Shedding light on plant competition: Modelling the influence of plant morphology on light capture (and vice versa). J. Theor. Biol. 2007, 244, 208-217. [CrossRef]

3. Watanabe, T.; Hanan, J.S.; Room, P.M.; Hasegawa, T.; Nakagawa, H.; Takahashi, W. Rice morphogenesis and plant architecture: Measurement, specification and the reconstruction of structural development by 3D architectural modelling. Ann. Bot. 2005, 95, 1131-1143. [CrossRef] [PubMed]

4. Carolan, M. Publicising food: Big data, precision agriculture, and co-experimental techniques of addition. Sociol. Rural. 2015, 57, 135-154. [CrossRef]

5. Afonnikov, D.A.; Genaev, M.A.; Doroshkov, A.V.; Komyshev, E.G.; Pshenichnikova, T.A. Methods of high-throughput plant phenotyping for large-scale breeding and genetic experiments. Russ. J. Genet. 2016, 52, 688-701. [CrossRef]

6. Pantalião, G.F.; Narciso, M.; Guimarães, C.; Castro, A.; Colombari, J.M.; Breseghello, F.; Rodrigues, L.; Vianello, R.P.; Borba, T.O.; Brondani, C. Genome wide association study (GWAS) for grain yield in rice cultivated under water deficit. Genetica 2016, 144, 651-664. [CrossRef]

7. Perez-Sanz, F.; Navarro, P.J.; Egea-Cortines, M. Plant phenomics: An overview of image acquisition technologies and image data analysis algorithms. Gigascience 2017, 6, gix092. [CrossRef]

8. Qiu, R.; Wei, S.; Zhang, M.; Li, H.; Sun, H.; Liu, G.; Li, M. Sensors for measuring plant phenotyping: A review. Int. J. Agric. Biol. Eng. 2018, 11, 1-17. [CrossRef]

9. An, N.; Palmer, C.M.; Baker, R.L.; Markelz, R.J.C.; Ta, J.; Covington, M.F.; Maloof, J.N.; Welch, S.M.; Weinig, C. Plant highthroughput phenotyping using photogrammetry and imaging techniques to measure leaf length and rosette area. Comput. Electron. Agric. 2016, 127, 376-394. [CrossRef]

10. Dimitrios, F.; Christoph, B.; Johannes, F.M.; Silke, K.; Alexander, P.; Fabio, F.; Andreas, U.; Ulrich, S. Rapid determination of leaf area and plant height by using light curtain arrays in four species with contrasting shoot architecture. Plant Methods 2014, 10, 9-21. [CrossRef]

11. Pereyrairujo, G.A.; Gasco, E.D.; Peirone, L.S.; Lan, A. GlyPh: A low-cost platform for phenotyping plant growth and water use. Funct. Plant Biol. 2012, 39, 905-913. [CrossRef]

12. Biskup, B.; Scharr, H.; Schurr, U.; Rascher, U. A stereo imaging system for measuring structural parameters of plant canopies. Plant. Cell Environ. 2007, 30, 1299-1308. [CrossRef]

13. Gibbs, J.A.; Pound, M.; French, A.P.; Wells, D.M.; Murchie, E.; Pridmore, T. Approaches to three-dimensional reconstruction of plant shoot topology and geometry. Funct. Plant Biol. 2016, 44, 62-75. [CrossRef]

14. Kjaer, K.H.; Ottosen, C.O. 3D laser triangulation for plant phenotyping in challenging environments. Sensors 2015, 15, 13533-13547. [CrossRef] [PubMed]

15. Xia, C.; Wang, L.; Chung, B.K.; Lee, J.M. In situ 3D segmentation of individual plant leaves using a RGB-D camera for agricultural automation. Sensors 2015, 15, 20463-20479. [CrossRef]

16. Drapikowski, P.; Kazimierczak-Grygiel, E.; Korecki, D.; Wiland-Szymańska, J. Verification of geometric model-based plant phenotyping methods for studies of xerophytic plants. Sensors 2016, 16, 924. [CrossRef]

17. Martínezguanter, J.; Garridoizard, M.; Valero, C.; Slaughter, D.C.; Pérezruiz, M. Optical sensing to determine tomato plant spacing for precise agrochemical application: Two scenarios. Sensors 2017, 17, 1096. [CrossRef]

18. Dionisio, A.; Mikel, C.; César, F.Q.; Ángela, R.; José, D. Three-dimensional modeling of weed plants using low-cost photogrammetry. Sensors 2018, 18, 1077. [CrossRef]

19. Paulus, S. Measuring crops in 3D: Using geometry for plant phenotyping. Plant Methods 2019, 15, 103-119. [CrossRef] [PubMed]

20. Thyagharajan, K.K.; Raji, I.K. A review of visual descriptors and classification techniques used in leaf species identification. Arch. Comput. Methods Eng. 2018, 1-28. [CrossRef]

21. Tan, J.W.; Chang, S.W.; Sameem, B.A.K.; Jen, Y.H.; Yong, K.T. Deep learning for plant species classification using leaf vein morphometric. IEEE/ACM Trans. Comput. Biol. Bioinform. 2018, 17, 82-90. [CrossRef]

22. Hang, L.; Tang, L.; Steven, W.; Yu, M. A robotic platform for corn seedling morphological traits characterization. Sensors 2017, 17, 2082. [CrossRef]

23. Chaivivatrakul, S.; Tang, L.; Dailey, M.N.; Nakarmi, A.D. Automatic morphological trait characterization for corn plants via 3D holographic reconstruction. Comput. Electron. Agric. 2014, 109, 109-123. [CrossRef]

24. Zermas, D.; Morellas, V.; Mulla, D.; Papanikolopoulos, N. 3D model processing for high throughput phenotype extraction-The case of corn. Comput. Electron. Agric. 2019, 172, 105047-105066. [CrossRef]

25. Li, X.; Wang, X.; Wei, H.; Zhu, X.; Huang, H. A technique system for the measurement, reconstruction and character extraction of rice plant architecture. PLoS ONE 2017, 12, e0177205. [CrossRef]

26. Fang, W.; Feng, H.; Yang, W.; Duan, L.; Chen, G.; Xiong, L.; Liu, Q. High-throughput volumetric reconstruction for 3D wheat plant architecture studies. J. Innov. Opt. Health Sci. 2016, 9, 1650037-1650050. [CrossRef]

27. Li, D.; Yan, C.; Tang, X.S.; Yan, S.; Xin, C. Leaf segmentation on dense plant point clouds with facet region growing. Sensors 2018, 18, 3625. [CrossRef] 
28. Kazmi, W.; Foix, S.; Alenya, G.; Andersen, H.J. Indoor and outdoor depth imaging of leaves with time-of-flight and stereo vision sensors: Analysis and comparison. Isprs J. Photogramm. Remote Sens. 2014, 88, 128-146. [CrossRef]

29. Yang, Z.; Han, Y. A low-cost 3D phenotype measurement method of leafy vegetables using video recordings from smartphones. Sensors 2020, 20, 6068. [CrossRef] [PubMed]

30. Li, D.; Cao, Y.; Shi, G.; Cai, X.; Chen, Y.; Wang, S.; Yan, S. An overlapping-free leaf segmentation method for plant point clouds. IEEE Access 2019, 7, 129054-129070. [CrossRef]

31. Schnabel, R.; Wahl, R.; Klein, R. Efficient RANSAC for point-cloud shape detection. Comput. Graph. Forum 2007, 26, 214-226. [CrossRef]

32. Mayer, D.G.; Butler, D.G. Statistical validation. Ecol. Model. 1993, 68, 21-32. [CrossRef]

33. Suresh, T.; Zhu, F.; Harkamal, W.; Yu, H.; Ge, Y. A novel LIDAR-based instrument for high-throughput, 3D measurement of morphological traits in maize and sorghum. Sensors 2018, 18, 1187. [CrossRef]

34. Ten Harkel, J.; Bartholomeus, H.; Kooistra, L. Biomass and crop height estimation of different crops using UVA-based lidar. Remote Sens. 2019, 12, 17. [CrossRef]

35. Xiaodan, M.; Kexin, Z.; Haiou, G.; Jiarui, F.; Song, Y. Calculation method for phenotypic traits based on the 3D reconstruction of maize canopies. Sensors 2019, 19, 1201. [CrossRef] 